\title{
Monoclonal antibodies directed to CD20 and HLA-DR can elicit homotypic adhesion followed by lysosome-mediated cell death in human lymphoma and leukemia cells
}

\author{
Andrei Ivanov, 1 Stephen A. Beers, ${ }^{2}$ Claire A. Walshe,2 Jamie Honeychurch, ${ }^{1}$ Waleed Alduaij, 1
} Kerry L. Cox, ${ }^{2}$ Kathleen N. Potter, ${ }^{2}$ Stephen Murray, ${ }^{1}$ Claude H.T. Chan, ${ }^{2}$ Tetyana Klymenko, ${ }^{1}$ Jekaterina Erenpreisa, ${ }^{3}$ Martin J. Glennie,2 Tim M. Illidge, ${ }^{1}$ and Mark S. Cragg²

${ }^{1}$ CRUK Paterson Institute for Cancer Research, School of Cancer and Imaging Sciences, School of Medicine, University of Manchester, Manchester, United Kingdom. ${ }^{2}$ Cancer Sciences Division, Southampton University School of Medicine, General Hospital, Southampton, United Kingdom. ${ }^{3}$ Biomedical Research and Study Centre, Riga, Latvia.

\begin{abstract}
mAbs are becoming increasingly utilized in the treatment of lymphoid disorders. Although Fc-Fc $\gamma \mathrm{R}$ interactions are thought to account for much of their therapeutic effect, this does not explain why certain $\mathrm{mAb}$ specificities are more potent than others. An additional effector mechanism underlying the action of some mAbs is the direct induction of cell death. Previously, we demonstrated that certain CD20-specific mAbs (which we termed type II mAbs) evoke a nonapoptotic mode of cell death that appears to be linked with the induction of homotypic adhesion. Here, we reveal that peripheral relocalization of actin is critical for the adhesion and cell death induced by both the type II CD20-specific mAb tositumomab and an HLA-DRspecific $\mathrm{mAb}$ in both human lymphoma cell lines and primary chronic lymphocytic leukemia cells. The cell death elicited was rapid, nonapoptotic, nonautophagic, and dependent on the integrity of plasma membrane cholesterol and activation of the V-type ATPase. This cytoplasmic cell death involved lysosomes, which swelled and then dispersed their contents, including cathepsin B, into the cytoplasm and surrounding environment. The resulting loss of plasma membrane integrity occurred independently of caspases and was not controlled by Bcl-2. These experiments provide what we believe to be new insights into the mechanisms by which 2 clinically relevant $m$ Abs elicit cell death and show that this homotypic adhesion-related cell death occurs through a lysosome-dependent pathway.
\end{abstract}

\section{Introduction}

$\mathrm{mAbs}$ are becoming increasingly utilized in the treatment of lymphoid disorders $(1,2)$. In particular, mAb directed to cell-surface antigens on malignant $B$ cells has proven the most clinically effective, with the anti-CD20 mAb, rituximab, being the first to be approved by the US FDA for the treatment of cancer. Rituximab has substantially improved outcome for patients with many different types of non-Hodgkin lymphoma and has now been administered to over 1 million patients in the decade since its approval. Despite such success, treatment is not curative and there is intense preclinical and clinical investigation of many other engineered mAbs directed to both CD20 and a host of other cell-surface antigens (2).

Authorship note: Andrei Ivanov and Stephen A. Beers are co-first authors and contributed equally to this work. Tim M. Illidge and Mark S. Cragg are co-senior authors and contributed equally to this work.

Conflict of interest: The authors have declared that no conflict of interest exists. Nonstandard abbreviations used: ADCC, Ab-dependent cellular cytotoxicity; BCR, $\mathrm{B}$ cell receptor; CDC, complement-dependent cytotoxicity; CLL, chronic lymphocytic leukemia; CTB, cholera toxin B subunit; EM, electron microscopy; FRAP, fluorescence recovery after photobleaching; HA, homotypic adhesion; LAMP-1, lysosomal-associated membrane protein 1; LFA-1, lymphocyte function-associated antigen-1; LMP, lysosome membrane permeabilization; MCD, methyl- $\beta$-cyclodextrin; $\mathrm{PCD}$, programmed cell death; QVD-OPH, quinoline-Val-Asp-difluorophenoxymethylketone; TEM, transmission EM; TPCK, chymotrypsin-like serine protease; V-ATPase, vacuolar ATPase; YFP, yellow fluorescent protein.

Citation for this article: J. Clin. Invest. 119:2143-2159 (2009). doi:10.1172/JCI37884.
The current challenge in the development of novel anti-cancer $\mathrm{mAbs}$ is to discover cell-surface antigens that will lead to efficient tumor cell killing and to identify biological pathways that will augment the cytotoxic effects of these mAbs. Central to this task is the need to identify the critical effector mechanisms involved in $\mathrm{mAb}$ therapy. Although $\mathrm{Fc}-\mathrm{Fc} \gamma \mathrm{R}$ interactions are thought to explain much of the therapeutic effect of mAbs, this does not explain why certain $\mathrm{mAb}$ specificities are more potent than others. In addition to "classical" Fc-dependent effector mechanisms such as complement-dependent cytotoxicity (CDC) and Ab-dependent cellular cytotoxicity (ADCC), certain mAbs are also able to trigger intracellular signaling in the target cell and directly induce programmed cell death (PCD) (reviewed in refs. 3, 4). Whereas ADCC and $\mathrm{CDC}$ are dependent only upon the level of surface expression and degree of modulation of a target molecule, PCD is reliant upon the nature of the target molecule with its associated signal transduction cascade. An enhanced understanding of how different mAbs evoke PCD is clearly central to providing new insights regarding not only their mode of action, but also how they might function in vivo and how mAb efficacy might be augmented.

We previously defined anti-CD20 mAbs as either type I or II, based upon their ability to redistribute CD20 into plasma membrane lipid rafts and their potency in various assays measuring CDC, homotypic adhesion (HA), and PCD (5-7). These experiments indicated that type II mAbs (such as tositumomab/B1), 
with their greater tendency to promote PCD but not CDC, are more effective at depleting malignant B cells in xenograft models (6). We have recently confirmed that this superior efficacy also translates to syngeneic models of B cell depletion in human CD20 $\mathrm{Tg}$ mice (8). In both cases, this enhanced activity was independent of the need for complement $(6,8)$ and no differences in ADCC (6) or macrophage uptake were apparent (8). Therefore, in the absence of other apparent effector mechanisms, it is possible that the enhanced induction of PCD may contribute to the greater efficacy of type II reagents. Previously, we demonstrated that the cell death induced by these type II mAbs was nonapoptotic, independent of caspase activity, and correlated with HA (5).

The induction of physiological or cytotoxic responses by extracellular signals requires the spatial and temporal coordination of distinct signaling pathways. Actin cytoskeleton remodeling has been established as an integrating mechanism in lymphocyte activation and antigen presentation, establishing immunological synapses and kinapses (reviewed in ref. 9). Disruption of the actin cytoskeleton inhibits both HA and cell death induced by antiHLA-DR $(10,11)$ and anti-CD99 Abs $(12,13)$. Moreover, this form of cell death, like that induced by type II anti-CD20 mAbs, is independent of caspase activation (12). HA has also been reported for B cells following the ligation of CD19, CD20, CD39, CD40 (10), and CD53 (14). Therefore, we hypothesized that cell signaling events leading to anti-CD20 mAb-induced HA and death might be linked through actin signaling.

Here, we show that actin redistribution toward the cell-tocell contact area is critical for both the adhesion and cell death induced with type II anti-CD20 mAbs and HLA-DR class II mAbs on a variety of cell lines and primary chronic lymphocytic leukemia (CLL) patient samples. The importance of the adhesion for efficient cell death is shown in experiments where cell contact is prevented. Furthermore, we demonstrate that actin dynamics are altered rapidly after $\mathrm{mAb}$ stimulation and that trogocytosis involving membrane swapping is stimulated. Actin redistribution is associated with structural changes in lysosomes that appear to swell and then disperse their contents, such as cathepsin B, into the cytoplasm, resulting in a loss of plasma membrane integrity and nonapoptotic cell death.

\section{Results}

Actin reorganization is involved in anti-CD20 and anti-HLA-DR-induced $H A$ and cell death. Following our earlier observation that HA correlates with cell death induced by anti-CD20 mAbs (5), we performed experiments to determine whether other $\mathrm{mAb}$ specificities were able to perform both of these functions. These experiments revealed that in addition to type II anti-CD20 reagents, mAbs to HLA-DR but not to HLA-DQ, HLA-DP, CD37, CD38, or B cell receptor $(\mathrm{BCR})$ were able to evoke both strong $\mathrm{HA}$ and cell death in Raji cells (Figure 1, A, B, and D, and data not shown). Death observed after 24 hours also correlated with a loss of clonogenic survival ( $\sim 8$-fold for tositumomab, a type II anti-CD20 mAb, and $\sim 80$-fold for L243 anti-HLA-DR) (data not shown). We concluded that the 2 processes of HA and cell death might be functionally linked. HA would require ultrastructural reorganization of the actin cytoskeleton, and so we first investigated the effects of blocking this activity on HA and cell death. These experiments, using agents that inhibit actin polymerization (cytochalasin D, latrunculin B), demonstrate a clear dependence of HA and cell death on actin reorganization (Figure 1 C, D, and E, and Supplemental
Figure 1; supplemental material available online with this article; doi:10.1172/JCI37884DS1). Furthermore, longer term cell-growth assays revealed that cotreatment with cytochalasin $\mathrm{D}$ was able to protect cells from $\mathrm{mAb}$ treatment for at least 72 hours (Figure 1D). To verify that cell-cell contact was important for cell death, we added low-melting point agarose to the cultures rapidly after addition of mAbs to prevent cell aggregation and then assessed cell death microscopically using SYTOX Green. SYTOX Green only fluoresces in dead cells, and these data clearly demonstrate that prevention of cell-cell aggregation severely limits cell death induction (Figure 2A). In contrast, delaying the addition of agarose until 1 hour after treatment with the mAbs failed to prevent either cell clustering or death. To confirm that these findings had relevance to primary tumors, we next investigated a panel of 7 CLL samples in these assays with largely similar results (Figure 2, B and C). These data show that both tositumomab and L243 mAbs evoked HA (Figure $2 \mathrm{~B}$ ) and increased cell death (Figure 2C) in an actin-dependent manner, inhibited by latrunculin $\mathrm{B}$, which itself resulted in a small $(\sim 5 \%)$ inhibitory effect on spontaneous cell death.

Previously, we have demonstrated that type I (but not type II) antiCD20 mAbs such as rituximab transduce many of their intracellular signals by "hijacking" the signaling capacity of the BCR (15). Therefore, given the similarity in cell death characteristics evoked by tositumomab and L243, we explored whether tositumomab might elicit cell death by interacting with HLA-DR molecules using the HLA-negative Raji variant (RJ.2.2.5). These cells lacked HLA-DR but expressed equivalent levels of CD20 compared with WT Raji cells and remained sensitive to cell death evoked by tositumomab (data not shown), demonstrating that tositumomab does not require HLA-DR expression for the induction of cell death.

Cholesterol-rich plasma membrane rafts are important for Ab-induced $H A$ and cell death. In order to investigate the pattern of cell-to-cell contact in cells undergoing HA after treatment with mAbs, we performed electron microscopy (EM) on Raji and SU-DHL4 cells. Ultrastructural studies of cells undergoing the early stages of HA revealed that the primary contact between cells was established by microvilli (Figure 3A). It has previously been reported that microvilli on B lymphocytes are enriched with detergent insoluble membrane rafts (16) containing CD20 (17) and are important for heterotypic adhesion during antigen presentation (16). We therefore assessed the impact of plasma membrane cholesterol on HA induced by $\mathrm{mAb}$ ligation of CD20 or HLA-DR surface antigens. Treatment of cells with methyl- $\beta$-cyclodextrin (MCD) resulted in a prominent decrease in the amount of plasma membrane cholesterol as detected by flow cytometry with FITC-labeled cholera toxin B subunit (CTB) (Figure $3 \mathrm{~B}$ ). No HA was observed in $\mathrm{mAb}$ treated cells that were pretreated with $\mathrm{MCD}$, whereas reincubation of cells with $\mathrm{MCD} /$ cholesterol complex to replete plasma membrane cholesterol restored $\mathrm{Ab}$-induced HA (Figure $3 \mathrm{C}$ ). To test the effect of cholesterol depletion on the cell death response, Raji cells were incubated in the presence or absence of MCD, with some samples replenished by incubation with $\mathrm{MCD} /$ cholesterol. The cells were further cultured in the presence of anti-CD20 (tositumomab or rituximab), anti-HLA-DR (L243) mAbs, or HBSS (to evoke nutrient and serum starvation) for 4 hours, and then cell death was assessed. MCD pretreatment attenuated cell death in both tositumomab-and L2 $43 \mathrm{mAb}$-treated samples but enhanced cell death in the HBSS-treated sample (Figure 3D). MCD-treated samples that were cholesterol replenished exhibited the same level of cell death as non-MCD-treated cells (Figure 3D). Importantly, 


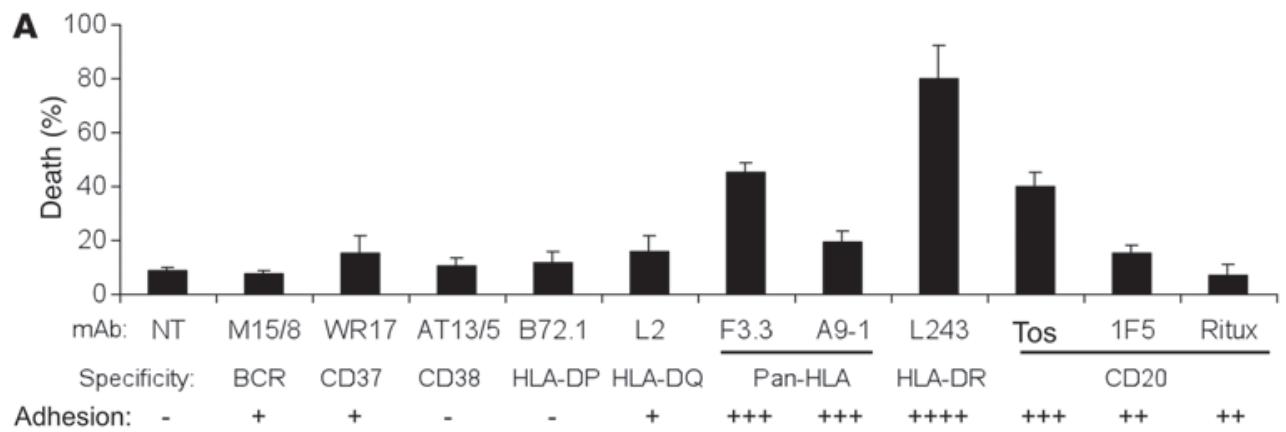

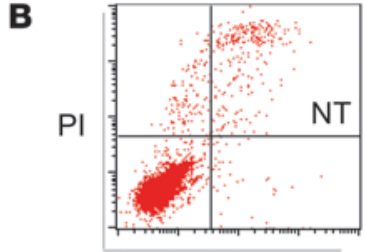

AnV-FITC

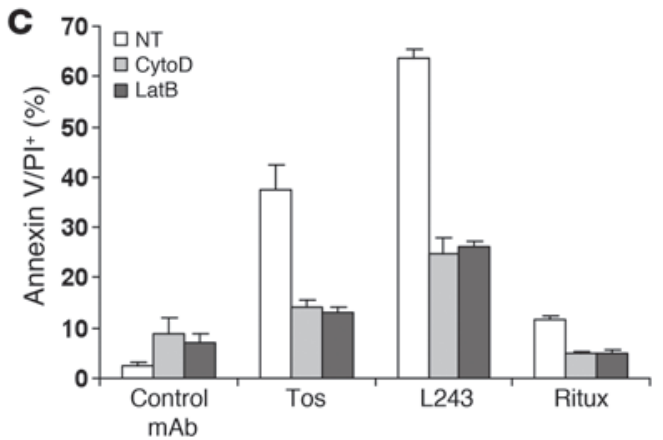

E

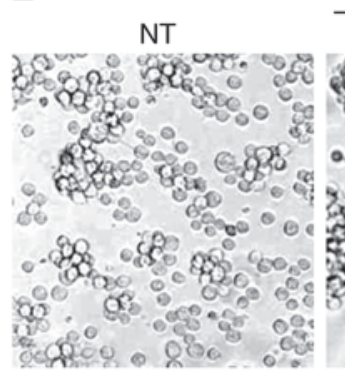

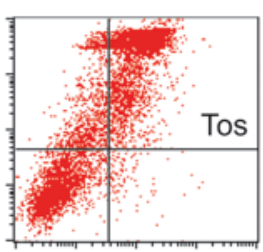
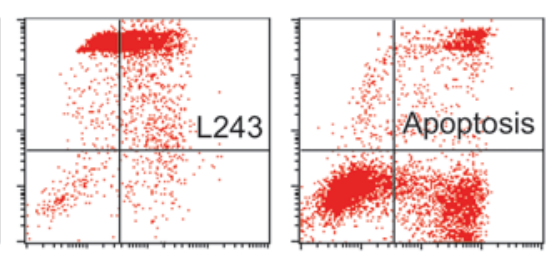

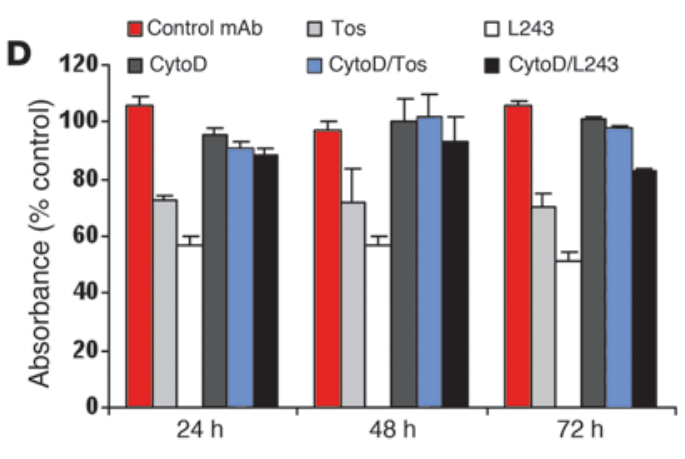

DMSO Tos
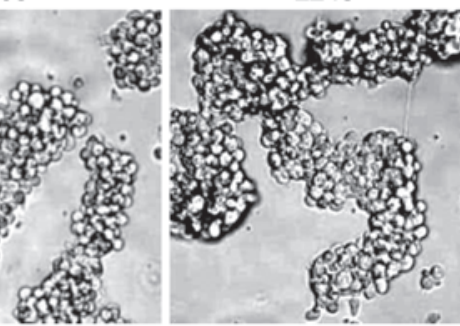

LatB

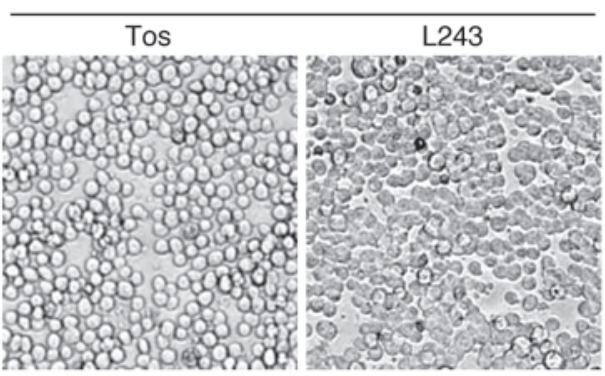

\section{Figure 1}

Actin-dependent HA is involved in cell death evoked by mAbs directed against CD20 or HLA-DR. (A) Raji cells were incubated with various $\mathrm{mAbs}(10 \mu \mathrm{g} / \mathrm{ml})$ for 4 to 6 hours, at which point HA was assessed by light microscopy. The number of plus signs indicates the strength/extent of adhesion as assessed semiquantitatively by microscopic visualization. 20 hours later, samples were assessed for the extent of cell death following staining with AnV-FITC (AnV) and propidium iodide (PI) and flow cytometry. Bars represent the mean cell death (AnV-and PI-positive cells) + SEM from 3 to 7 independent experiments. Representative data are shown in B. A typical "apoptotic" plot is shown for reference following treatment of Raji cells with 4 Gy irradiation. Tos, tositumomab; Ritux, rituximab; NT, no treatment. (C) Raji cells were treated with various actin inhibitors prior to the addition of anti-CD20 or HLA-DR mAbs, and cell death was assessed 4 hours later as previously described. (D) Inhibitor of actin cytoskeleton at noncytotoxic concentrations protects cells from long-term cytotoxicity evoked by both anti-CD20 and HLA-DR mAbs. Prior to the addition of various mAbs $(5 \mu \mathrm{g} / \mathrm{ml})$, Raji cells were treated for 45 minutes with cytochalasin $\mathrm{D}(\mathrm{CytoD} ; 0.1 \mu \mathrm{M})$. Cell viability was assessed 24, 48, and 72 hours later using Cell Proliferation Kit II (sodium 3'-[1-(phenylaminocarbonyl)-3,4-tetrazolium]-bis (4-methoxy-6-nitro) benzene sulfonic acid hydrate [XTT] assay; Roche). Plot represents mean metabolic activity relative to nontreated control from 2 independent experiments run in 3 technical replicates. Data shown represent mean + SEM. (E) Raji cells were treated with DMSO or the actin inhibitor latrunculin B (LatB; $10 \mu \mathrm{M})$ prior to the addition of Tos or L243 $(10 \mu \mathrm{g} / \mathrm{ml})$ and assessed for HA 4 to 6 hours later. Original magnification, $\times 20$. Together, these data clearly demonstrate that cell death evoked by both anti-CD20 and HLA-DR mAbs is dependent upon adhesion and that both these processes are dependent upon actin. 
A

Control mAb

Tos

L243
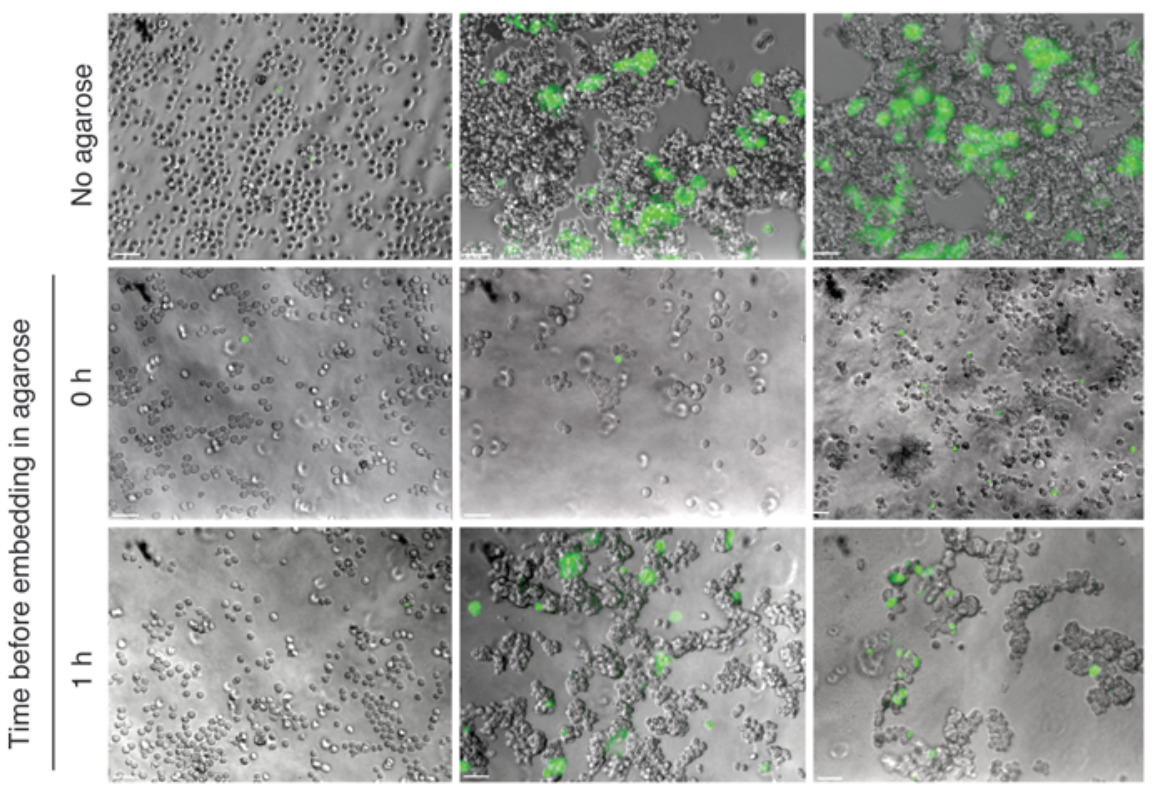

B

NT
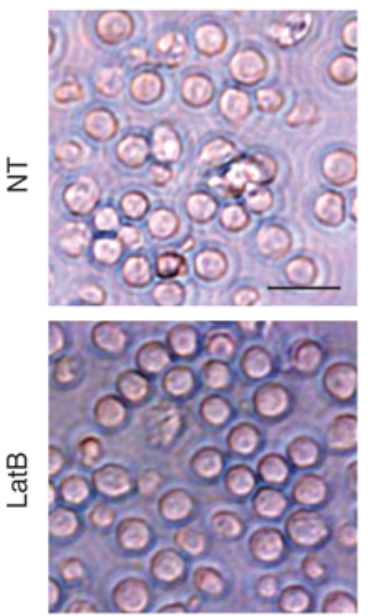

Tos
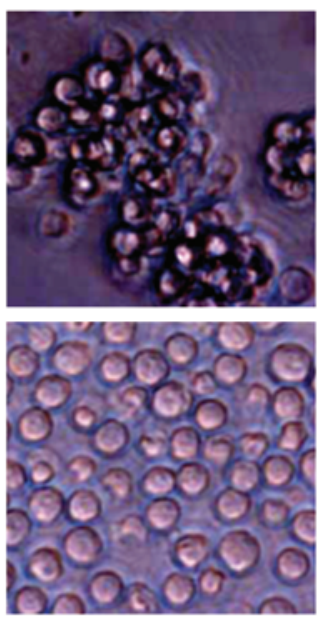

Ritux
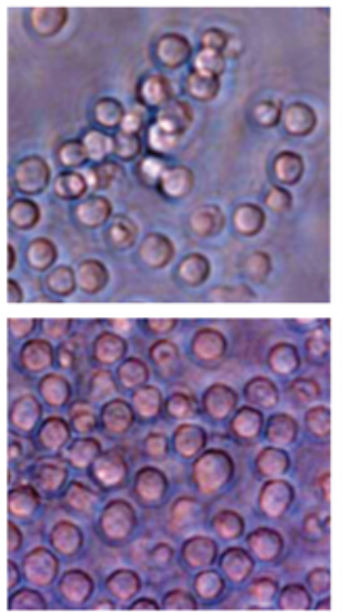

L243
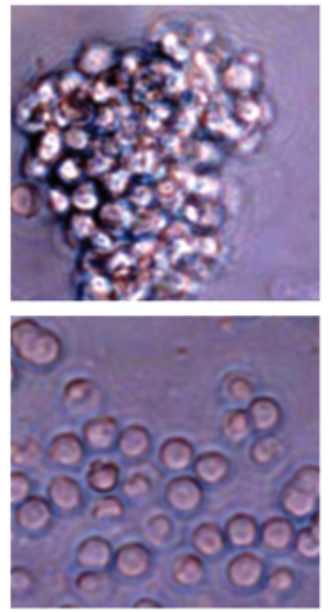

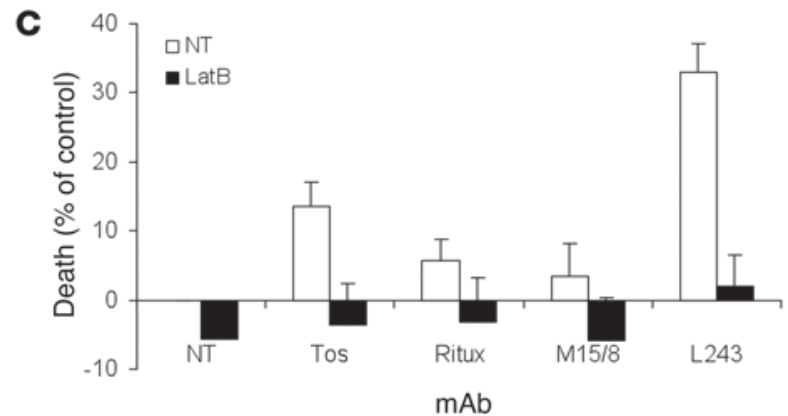

Figure 2

Direct involvement of HA in cell death evoked by CD20 and HLA-DR mAbs. (A) Cells were treated with tositumomab or L243 mAbs, plated as usual or embedded either immediately or 1 hour later in low-melting point agarose containing SYTOX Green nuclear stain to prevent cell-cell association, and assessed by fluorescence microscopy 4 hours after treatment. Scale bars: $100 \mu \mathrm{m}$. (B) CLL cells were treated or not with the actin inhibitor latrunculin $B(10 \mu \mathrm{M})$ for 45 minutes prior to the addition of various mAbs $(10 \mu \mathrm{g} / \mathrm{ml})$ for 18 hours, when HA was assessed by light microscopy. A typical example of the results is demonstrated. Scale bar: $40 \mu \mathrm{m}$. (C) The same samples were then assessed for the extent of cell death following staining with AnV-FITC and PI and flow cytometry. Bars represent the mean cell death (AnV-and PI-positive cells) above cell death observed in the untreated samples + SEM from 7 different CLL samples. Due to heterogeneous levels of basal apoptosis in the CLL samples, these data were expressed as percentage of cell death above control. These data clearly demonstrate that adhesion and death evoked by both anti-CD20 and HLA-DR mAbs in CLL samples are dependent upon actin. 
A
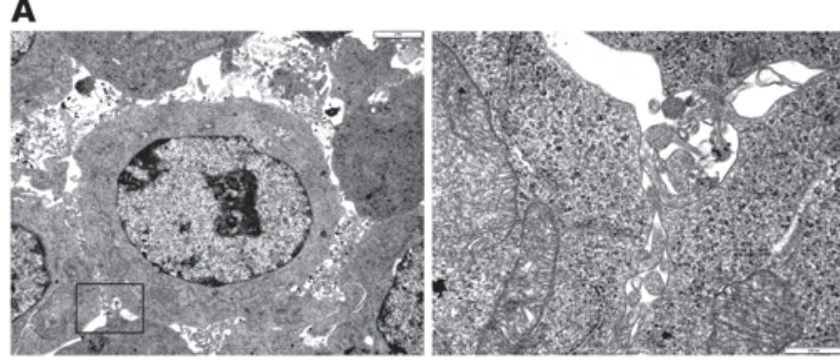

B Background

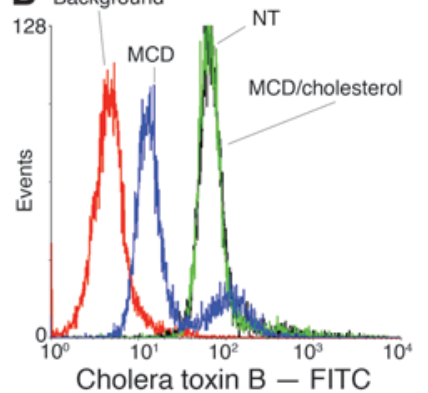

C MCD Cholesterol
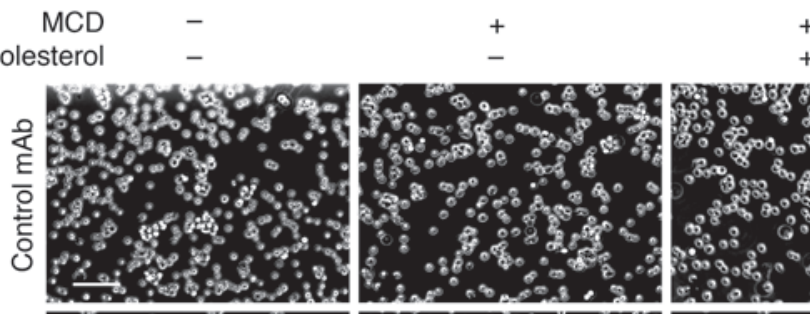

$+$
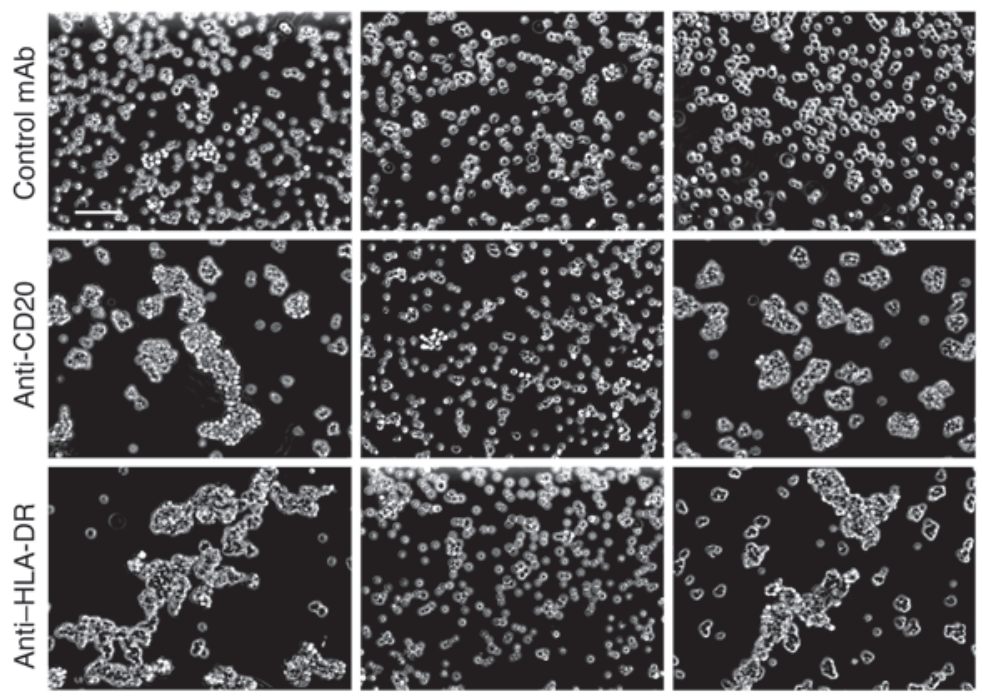

D
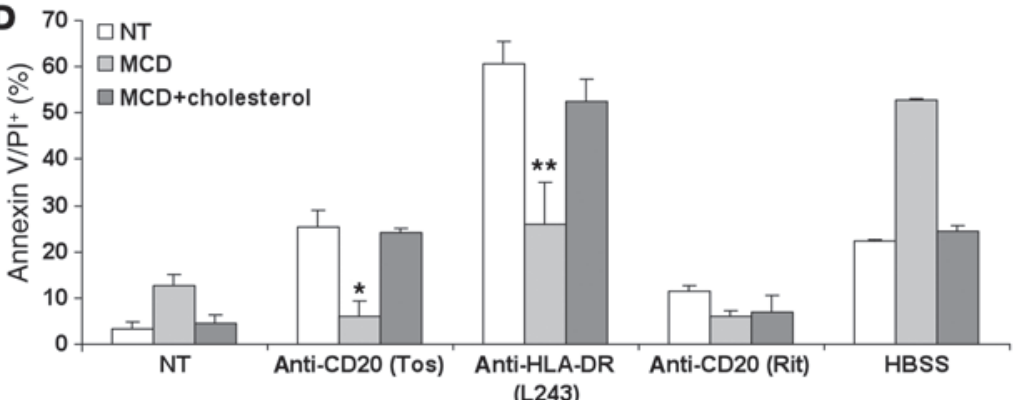

(L243)

Treatment

\section{Figure 3}

Involvement of microvilli and plasma membrane cholesterol in mAb-induced HA. (A) Raji cells were incubated with tositumomab or L243 Abs $(10 \mu \mathrm{g} / \mathrm{ml})$ for 1 hour. Then samples were collected and processed for TEM as described in Methods. Left panel shows the early stage of intercellular adhesion of Raji cells. Scale bar: $2 \mu \mathrm{m}$. Right panel shows high-power view of the area shown in the square demonstrating the involvement of microvilli in HA of Raji cells. Scale bar: $0.5 \mu \mathrm{m}$. (B) Assessment of plasma membrane cholesterol in MCD-pretreated Raji cells. MCDtreated cells were harvested and stained with FITC-labeled CTB and assessed by flow cytometry. The prominent reduction of plasma membrane cholesterol observed in MCD-treated cells (MCD) could be reversed by coincubation of cells with cholesterol (MCD/cholesterol). CTB unlabeled cells are shown as a background control (background). (C) Raji cells were pretreated with $10 \mu \mathrm{M}$ MCD for 30 minutes, then washed and incubated with tositumomab or L243 Abs $(10 \mu \mathrm{g} / \mathrm{ml})$ for 4 hours. Then aggregation of cells was assessed by inverted phase contrast microscopy. Scale bar: $150 \mu \mathrm{m}$. (D) Involvement of plasma membrane cholesterol in anti-CD20- and anti-HLA-DRinduced cell death. Plasma membrane cholesterol was modulated as described in $\mathbf{B}$ and $\mathbf{C}$ and then cells were incubated with Abs (10 $\mu \mathrm{g} / \mathrm{ml}$ ) or in HBSS. Cell death was assessed 4 hours later. Data represent the average of at least 2 independent experiments + SEM. ${ }^{\star} P<0.01 ;{ }^{* *} P<0.003$. the extraction of plasma membrane cholesterol did not alter the surface expression of CD20 or HLA-DR (Supplemental Figure 2), although it was able to completely block the HA triggered by mAbs to these antigens. Thus HA and cell death induced by both of these mAbs appear dependent upon membrane cholesterol, most likely through the interaction of microvilli.

Peripheral relocalization and increased lateral mobility of actin during $A b$-induced $H A$. After establishing the role of actin in cell death induced by anti-CD20 and HLA-DR mAbs, we next investigated actin dynamics after ligation of CD20 or HLA-DR. Using Alexa Fluor 584-labeled phalloidin, we studied the distribution of F-actin in Raji cells before and after treatment with anti-CD20 (Figure 4A) and anti-HLA-DR mAbs. These experiments revealed a clear cytoskeleton actin ring around nuclei in nontreated cells, while loss of its integrity accompanied by redistribution of F-actin to the areas of cell-cell contact was observed after treatment with both mAbs. To allow us to assess the kinetics of actin relocalization, we subsequently performed time-lapse microscopy of Raji cells transfected to express a GFP-actin fusion protein. The redistribution of actin toward cell-to-cell contact areas was also observed using this approach and started within 2 to 10 minutes, coinciding with the onset of HA, reaching completion/maximal adhesion in about 40 minutes (Figure 4B; Supplemental Video 1). Importantly, Western blotting analysis showed no increase in the total amount of actin during this period (Figure 4C), which is indicative of actin redistribution rather than additional de novo synthesis of G-actin. Furthermore, careful analysis of the actin staining pattern showed a decrease in the fluorescence signal area 

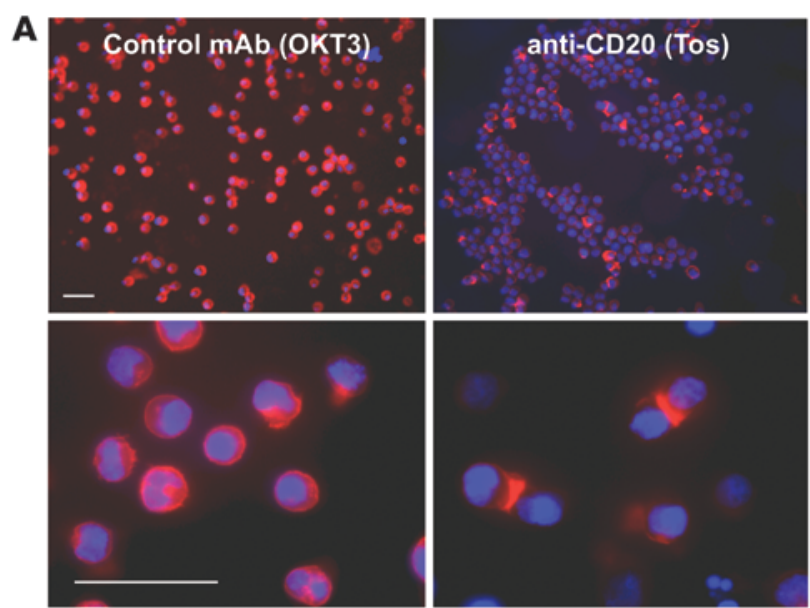

$\mathbf{B}$
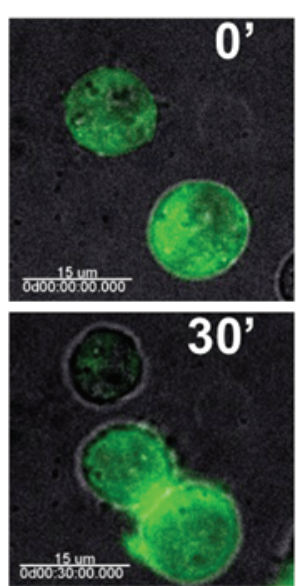
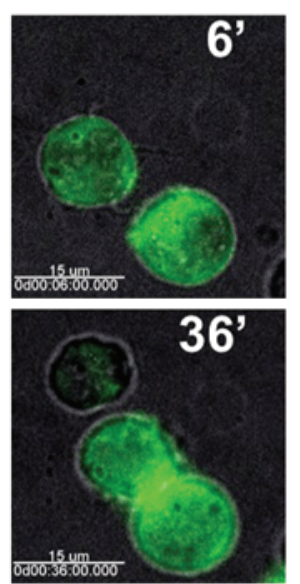
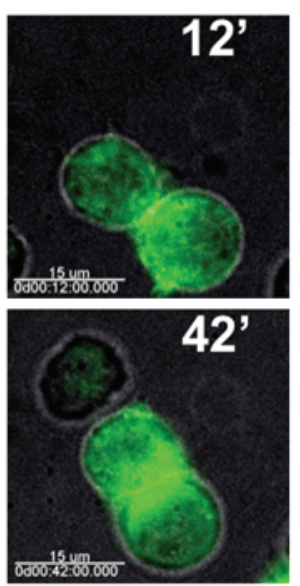

C MW $(\mathrm{kDa})$
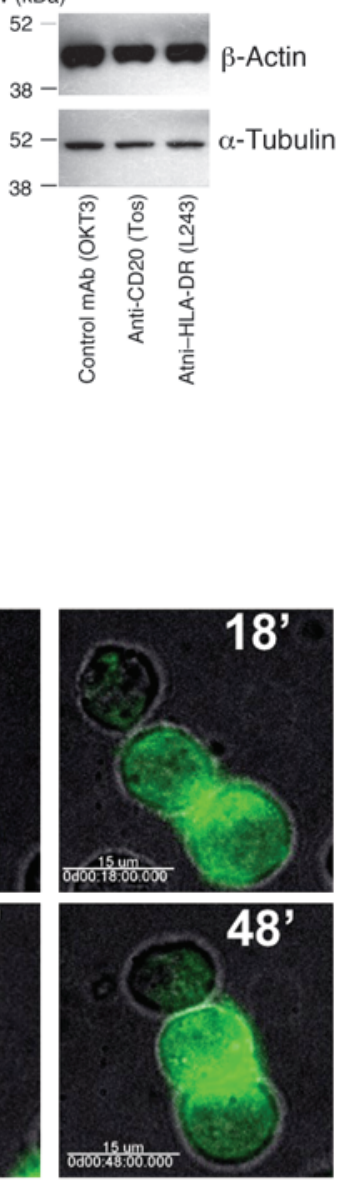
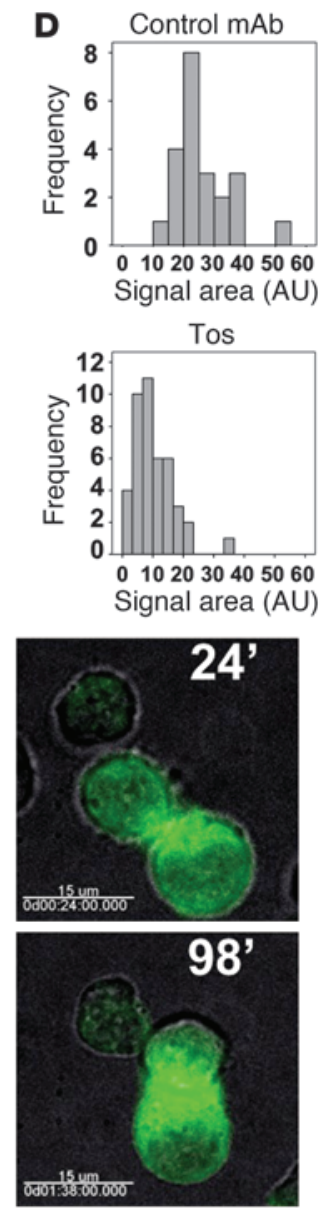

\section{Figure 4}

Peripheral relocalization of cellular actin in cells undergoing HA after treatment with tositumomab. (A) Raji cells were incubated with the anti-CD20 Ab tositumomab or L243 (10 $\mu \mathrm{g} / \mathrm{ml})$, washed, and sedimented onto poly-L-lysine-coated microscope slides. After fixation with $1 \%$ paraformaldehyde in PBS, cells were stained with Alexa Fluor 594-labeled phalloidin (red). DNA was counterstained with DAPI (blue). Lower panels represent higher-power images. Scale bars: $60 \mu \mathrm{m}$. (B) Time-lapse microscopy of Raji cells expressing AcGFP-labeled actin. After addition of tositumomab or L243 $(10 \mu \mathrm{g} / \mathrm{ml})$, cell suspensions were put into a glass-bottom Petri dish and assessed at $37^{\circ} \mathrm{C}$ under inverted timelapse microscopy. Images were obtained every 2 minutes (see Supplemental Video 1). The interval between images displayed is 6 minutes, except for between the final 2 images (50 minutes). Scale bars: $15 \mu \mathrm{m}$. (C) The total amount of cellular actin was assessed by Western blotting. $\alpha$-tubulin was assessed as a loading control. Samples were taken 24 hours after stimulation. (D) Image analysis of F-actin signal area following treatment with control mAbs (OKT3) or tositumomab. Cells were sedimented onto poly-L-lysine-coated slides and stained with phalloidin-Alexa Fluor 594; images were taken. The area of Alexa Fluor-positive signal was then measured using Image-Pro Plus software, version 6.3 (Media Cybernetics), and expressed graphically.

(Figure 4D), suggesting that compartmentalization rather than de novo synthesis of cellular F-actin is responsible for the increased actin signal at the cell-cell contact area.

Next, we performed fluorescence recovery after photobleaching (FRAP) analysis to investigate the mobility of actin after treatment of cells with anti-CD20 and HLA-DR mAbs. The speed of fluorescence signal recovery was measured at several time points after addition of mAbs (Figure 5, A and B, and Supplemental Videos 2-4). These data indicate that the recovery of actin fluorescence is much faster in cells treated with tositumomab or L243 mAbs than in control cells, in which no recovery could be observed within 100 seconds. Moreover, the faster recovery of fluorescence was evident in both cytoplasmic and peripheral compartments in cells treated with tositumomab, suggesting much faster overall turnover of F-actin in mAb-treated cells (data not shown). Importantly, all of the above measurements were performed in morphologically viable cells and no fluorescence recovery was detected in dead cells (data not shown). Furthermore, the recovery of the fluorescent actin signals returned to baseline levels or greater within the first 10 minutes after addition of mAbs (data not shown). This suggests that the log phase of actin redistribution occurs within minutes of mAb treatment.

Video microscopy analysis also revealed that although cell aggregation occurred within 5 minutes following addition of $\mathrm{mAbs}$, it remained stable for at least 48 hours (data not shown). Moreover, close observation of the cell-cell interactions during HA revealed the formation of small $(\sim 5-\mu \mathrm{m}$ diameter) transient cytoplasmic bridges between cells that opened and closed at approximately 5-minute intervals (Figure 5C).

Interestingly, cells treated with tositumomab or L243 mAbs also displayed peripheral relocalization of enlarged mitochon- 

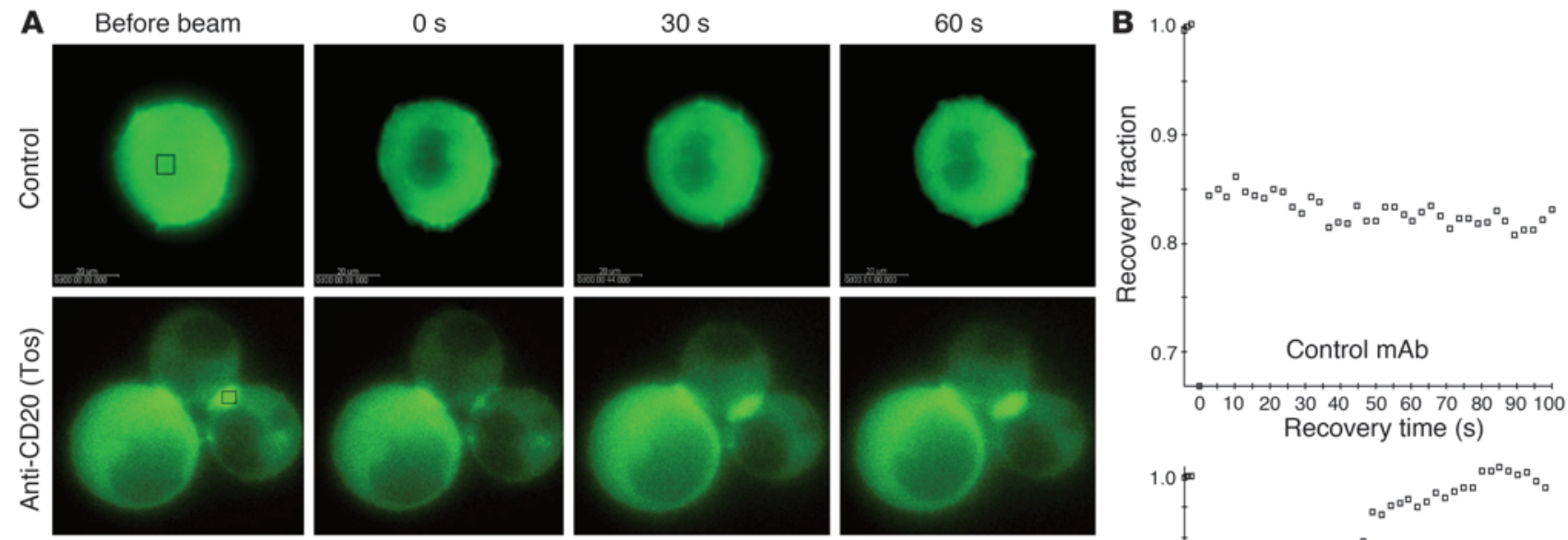

C

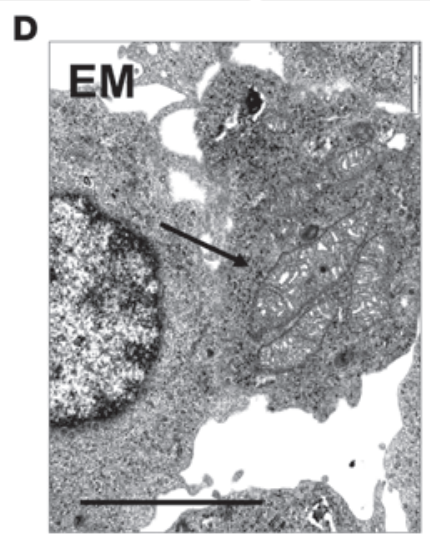

E
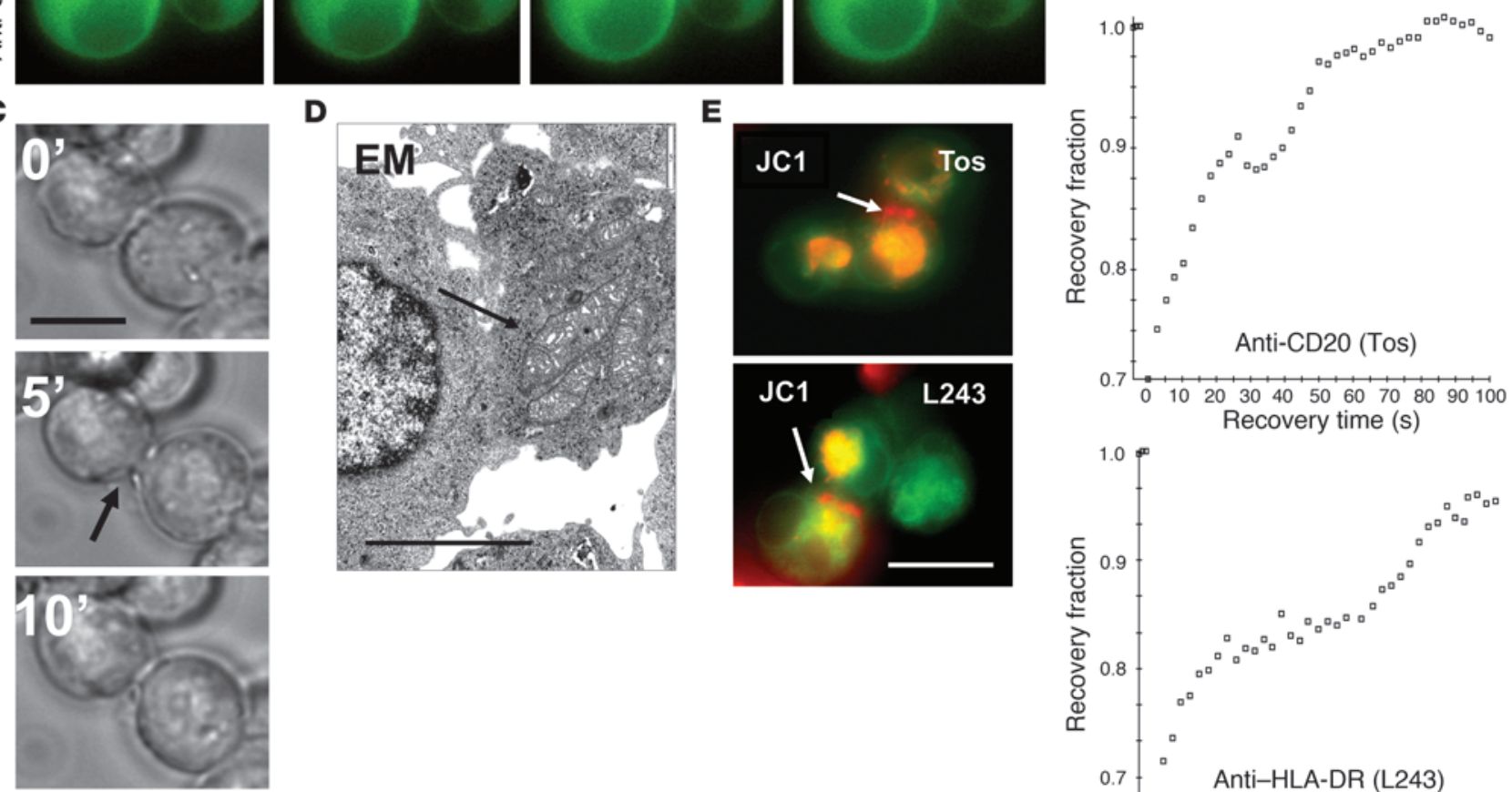

$\mathbf{F}$
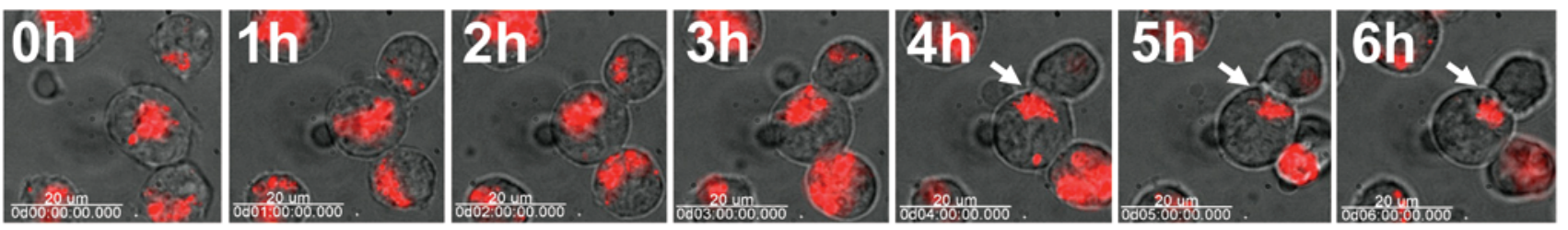

\section{Figure 5}

Lateral mobility of cellular actin, formation of syncytium, and polarization of mitochondria in cells undergoing HA. (A) AcGFP-actin-expressing Raji cells were treated with either control mAbs (OKT3), tositumomab, or L243 (all at $10 \mu \mathrm{g} / \mathrm{ml}$ ) for 4 hours, then studied by FRAP, with flurorescence recovery assessed for 100 seconds. Typical image data is shown for control- and tositumomab-treated cells. Upper panels: virtually no recovery was observed in control mAb-treated cells. Lower panels (Tos): recovery of junctional actin within the cell-to-cell contact area was observed after approximately 80 seconds. Scale bars: $20 \mu \mathrm{m}$. Squares indicate regions targeted for photobleaching. (B) Typical fluorescence recovery pattern of AcGFP-actin after photobleaching. Control cells showed no recovery within 100 seconds (left). Recovery of junctional actin fluorescence was shown within 80 seconds after photobleaching in cells treated with anti-CD20 (Tos; center) and anti-HLA-DR (L243; right). (C) Transient cytoplasmic bridges between cells undergoing HA. Phase contrast time-lapse microscopy of Raji cells treated with tositumomab (10 $\mu \mathrm{g} / \mathrm{ml}$ ). Intervals between images are 5 minutes. Arrows indicate location of bridge formation. Scale bar: $20 \mu \mathrm{m}$. (D and E) Peripheral relocalization of mitochondria toward cell-to-cell contact area as assessed by TEM (D) and JC-1 staining (E). Arrows indicate localized mitochondria. Scale bar: $10 \mu \mathrm{m}$. (E) JC-1-labeled cells were treated with tositumomab or L243 (10 $\mu \mathrm{g} / \mathrm{ml})$. After that, images were captured detecting monomeric (green) and J-aggregate (red) forms of JC-1. Arrows indicate J-aggregate mitochondria at cell-cell junctions. Scale bars: $10 \mu \mathrm{m}$ (D); $20 \mu \mathrm{m}$ (E). (F) Time-lapse video microscopy of JC-1-labeled cells. Red mitochondria represent mitochondria with high membrane potential, migrating toward cell-to-cell contact areas. Interval between images is 1 hour. Scale bar: $20 \mu \mathrm{m}$. 
dria toward the cell-to-cell contact areas (Figure 5, D and E). EM showed well-developed cristae in the juxtaposed mitochondria, suggesting that they are in an energy-producing phase (Figure 5D). Furthermore JC-1 staining for mitochondria revealed that only mitochondria maintaining high membrane potential, represented as J-aggregates of JC-1 dye, were moving toward the cell-to-cell contact area (Figure 5E), and this process occurred within approximately 3 hours of addition of mAbs (Figure 5F and Supplemental Video 5). Additionally, peripheral relocalization of mitochondria could be inhibited with the actin polymerization inhibitor latrunculin B (Supplemental Figure 3). These data indicate that cell-cell adhesion upon ligation of surface antigens may be an active process that requires energy from polarized mitochondria in situ that, in turn, are driven or immobilized by the actin cytoskeleton.

CD20-induced HA is accompanied by plasma membrane exchange and correlates with cell death. Having observed the apparent physical linkage between apposing cells participating in HA (Figure 5C), we hypothesized that mutual exchange of cellular components might occur among the cells undergoing HA. To address this hypothesis, we labeled Raji cells with the PKH26 cell membrane dye (red) and mixed them at a 1:1 ratio with Raji cells expressing a GFP-actin fusion protein (green). The cell mixture was treated with tositumomab or L243, and then the green and red fluorescence were assessed by fluorescence microscopy (Figure 6A) and flow cytometry (Figure 6B). Following control incubation in the absence of $\mathrm{mAb}$ stimulation for 24 hours, we observed that only a very low percentage $(<1 \%)$ of cells became double stained, indicating that spontaneous transfer of cellular material was a rare event. However, following stimulation with tositumomab or L243, PKH26-labeled membrane was taken up by $8 \%$ and $15 \%$ of GFP-actin-expressing cells, respectively (Figure 6B). Fluorescence microscopy indicated that actin patches were not detected in PKH26-labeled cells, suggesting that cells undergoing HA exchange membranes but not actin cytoskeletal components (Figure 6A). Next, we performed the same experiments mixing PKH26-labeled cells with cells expressing yellow fluorescent protein (YFP) in the cytosol. This confirmed earlier work, with approximately $8 \%$ of cells dual stained (PKH26 and YFP) 24 hours after tositumomab treatment (Figure 6B). Similar results were obtained when cells were incubated for shorter periods (2-4 hours; data not shown). Higher levels ( double) of membrane transfer were observed when transfer experiments were performed using 2 membrane dyes (PKH26 and PKH67), again indicating that only membrane components were being transferred among the cells after $\mathrm{mAb}$ stimulation (data not shown). To expand these observations, we then tested a larger panel of mAbs for their ability to induce intercell membrane exchange and determined whether there was a relationship between $\mathrm{mAb}$-induced membrane swap and cell death induction. Figure $6 \mathrm{C}$ clearly demonstrates that a correlation exists, as mAbs that induced membrane exchange were more efficacious in evoking cell death. However, subsequent annexin $\mathrm{V}-\mathrm{Cy} 5.5$ labeling indicated that equivalent numbers of cells were dying regardless of whether they had undergone membrane exchange (Figure 6D). Interestingly, Fc-Fc $\gamma \mathrm{R}$ interactions did not appear to influence either of these properties, as $\mathrm{F}(\mathrm{ab})_{2}{ }_{2}$ fragments of tositumomab and L243 elicited the same amount of cell death and membrane exchange as did whole IgG (Figure 6C). Having established the dynamics and critical role of actin in the cell death process engaged by both CD20 and HLA-DR mAbs, we next investigated more closely the nature of the cell death elicited by these mAbs.
Ab-induced cell death has a distinctive morphology and is neither apoptotic nor autophagic. We previously demonstrated that cell death evoked by type II CD20 mAbs was not blocked by overexpression of Bcl-2 in Ramos-EHRB cells (5) or Raji cells (18). Here, we performed similar experiments also assessing HLA-DR mAbs in Raji cells overexpressing Bcl-2. The data obtained 24 hours after treatment with mAbs confirm our previous observations and demonstrate that HLA-DR mAbs (unlike gamma radiation) (18) are also able to bypass the mitochondrial death pathway regulated by Bcl-2 (Figure 7A). Furthermore, inhibition of caspases using carbobenzoxy-valyl-alanyl-aspartyl-[O-methyl]-fluoromethylketone (ZVAD-FMK) (data not shown) or quinoline-Val-Asp-difluorophenoxymethylketone (QVD-OPH) had no effect on cell death. In contrast, caspase inhibition was able to block cell death elicited by mitoxantrone. Furthermore, the combination of caspase inhibition and $\mathrm{Bcl}-2$ overexpression was also unable to block the cell death evoked by either $\mathrm{mAb}$, confirming that this cell death was independent of typical apoptotic regulation (Figure 7A).

In the last 5 years, the number of nonapoptotic death pathways described in mammalian cells has steadily increased (19). Of those, perhaps the best characterized is that resulting from autophagy, which involves the formation of autophagosomes that are governed through the tightly regulated interactions of Atg proteins. Therefore, given the absence of an apoptotic death program, we next determined whether autophagy was elicited by CD20 and HLA-DR mAbs. First, we assessed the expression of several key Atg proteins before and after $\mathrm{mAb}$ treatment. These experiments revealed that Atg5, Atg12, and Beclin-1 were unchanged over the 24-hour time course studied (Figure 7B and data not shown). Moreover, 2 independent siRNAs capable of reducing Beclin-1, one of the key proteins implicated in autophagic death, had no effect on either the level of HA (data not shown) or cell death evoked by tositumomab or L243 (Figure 7C). As a control in these experiments, we also employed a nonfunctional siRNA targeted to Atg12, which had no effect on Beclin-1 or cell death. Furthermore, it appears that Raji cells are relatively resistant to autophagy induction per se, as classical inducers of autophagy such as rapamycin, hypoxia, nutrient and serum starvation, and combinations thereof were unable to induce changes in Atg5, Atg12, Beclin-1, or LC3 (Supplemental Figure 4 and data not shown).

Previous workers have indicated that nonapoptotic, nonautophagic type III death, such as evoked by CD47 ligation, results from activation of chymotrypsin-like serine proteases (TPCK) (20). To determine whether these enzymes were involved in the cell death evoked by tositumomab and L243, we examined the effects of previously characterized inhibitors of these enzymes in cell death assays. These experiments, detailed in Figure 7D, clearly demonstrate that cell death was not blocked by any of the inhibitors to TPCK or trypsin-like serine proteases, indicating that death engaged by tositumomab and L243 mAbs is independent of these enzymes.

Ultrastructural studies confirmed that neither classical apoptotic nor autophagic death was evoked with tositumomab or L243 mAbs (Figure 8). First, there was no evidence of DNA condensation or apoptotic body formation typical of apoptosis. Instead, transmission EM (TEM) revealed large cytoplasmic inclusions and vacuoles in cells (Figure 8, A and B) that were distinct from both control cells (Figure 8C) and cells undergoing classical apoptosis (Figure 8D). This pattern was coincident with cells displaying loss of microvilli (Figure 8E) and permeabilized plasma membranes (Figure 8F) while undergoing HA, as assessed by scanning EM (Fig- 

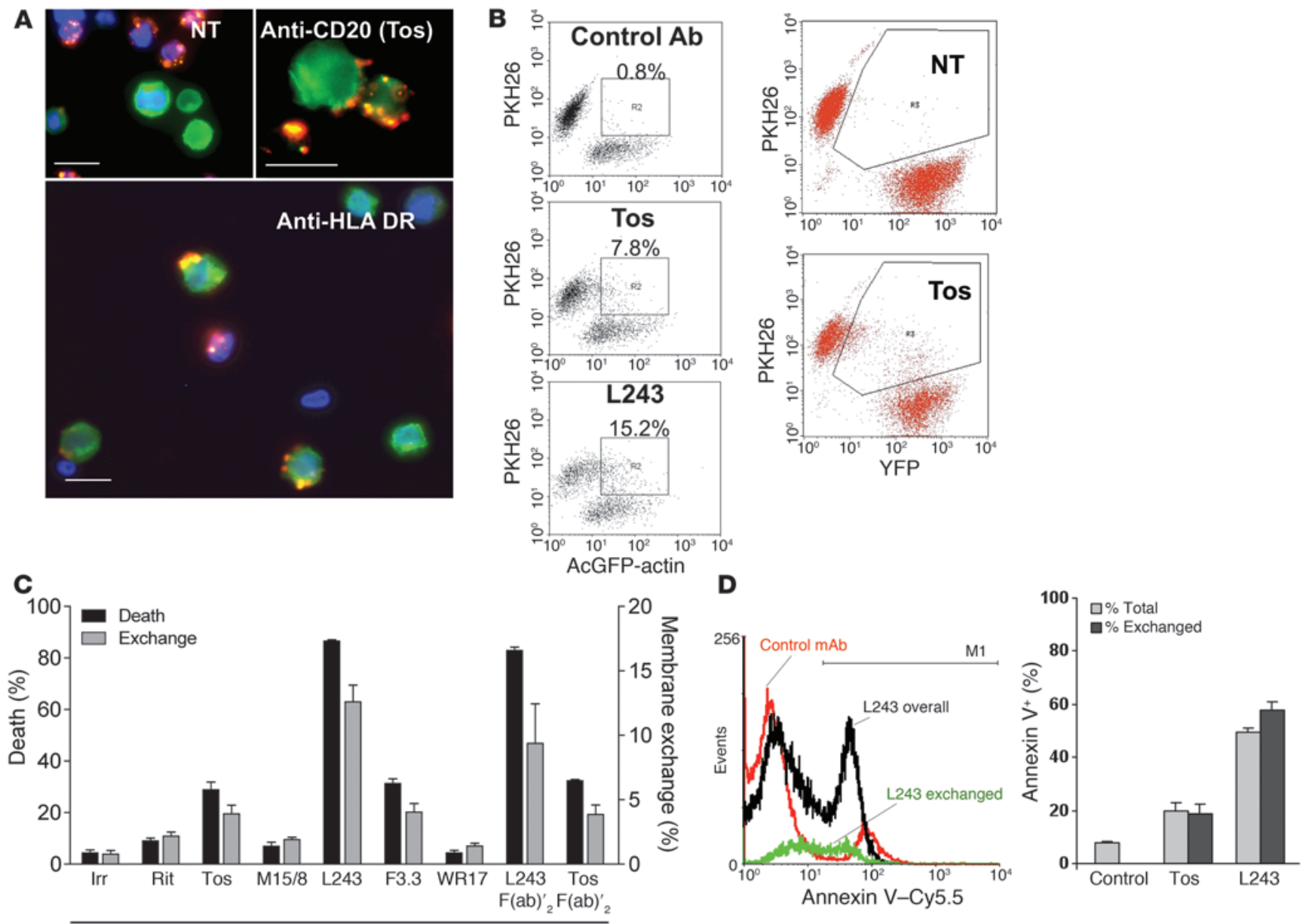

Treatment

\section{Figure 6}

CD20-induced HA is accompanied by plasma membrane exchange and correlates with cell death. (A) Raji cells were labeled with PKH26 (red), mixed with pGFP-actin-expressing (green) Raji cells, and treated with $10 \mu \mathrm{g} / \mathrm{ml}$ tositumomab or L243 and examined by fluorescence microscopy after 24 hours. Scale bars: $20 \mu \mathrm{m}$. (B) Flow cytometry profiles of membrane exchange using PKH26-labeled and YFP-expressing cells (right) and PKH26-labeled and GFP-actin-expressing cells (left) at 24 hours after treatment with tositumomab or L243. Values indicate the percentage of cells double positive for PKH26 and AcGFP. (C) Correlation between cell death and membrane exchange. The data clearly demonstrate that type II anti-CD20 and HLA-DR mAbs stimulate transfer of plasma membrane material between adhering cells whereas other mAbs do not. This property is independent of Fc-FcyR interactions, occurring equivalently with $\mathrm{F}(\mathrm{ab})^{\prime}{ }_{2}$ fragments, and was correlated with the extent of cell death induced. Data shown are mean + SEM. (D) Similar proportion of cell death in overall population and in cells having undergone plasma membrane exchange. Left panel: cell death was assessed by Cy5.5-labeled annexin V in FL4 channel of FACScalibur 4 hours after treatment with L243. The histogram represents the pattern of annexin V positivity (M1 region) in the overall cell population (black) and in the population of dual-positive cells (PKH26/PKH67; green). Red histogram shows control-treated cells. Right panel: numerical data representing the proportion of cell death in dually PKH26/PKH67-positive cells and in the overall cell population in 2 independent experiments + SEM. The data show no enrichment of cell death in the cells that have undergone membrane exchange.

ure 8, G and H). Again, this morphological pattern of cell death differed significantly from what was seen in control cells (Figure 8I) and cells undergoing classical apoptosis (Figure 8J). Although vacuoles were evident, these were not autophagic in nature, as they lacked double-membrane structure - the hallmark of autophagy. Rather, our findings are most in accordance with the morphological criteria described for cells said to be undergoing cytoplasmic cell death (21-23). Indeed, the proportion of cells with decomposing cytoplasm (as in Figure 8, A and B) was approximately $20 \%$ and $70 \%$ following treatment with tositumomab or L243, respectively, and roughly equivalent to the proportion of cell death following these treatments. In summary, these data indicate that cell death arising after engagement of CD20 or HLA-DR by mAbs is not evoked through conventional apoptotic or autophagic routes but instead appears to involve a "cytoplasmic" death pathway.

Involvement of lysosomes in cell death evoked by tositumomab or L243. It is well established that lysosomes play important roles in nonapoptotic cell death (24), and it has even been proposed that the lysosome is the primary organelle responsible for cell death triggered by engagement of the BCR for antigen (25). Enlargement of the lysosomal compartment and subsequent lysosome membrane permeabilization (LMP) are hallmarks of cytoplasmic cell death (26). 


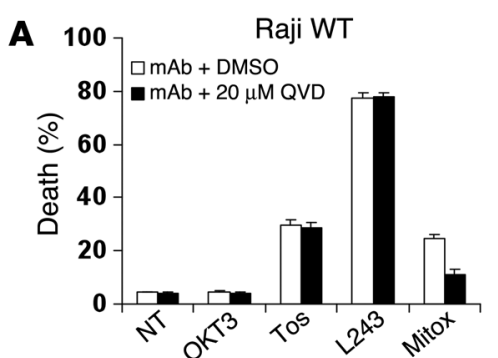

B
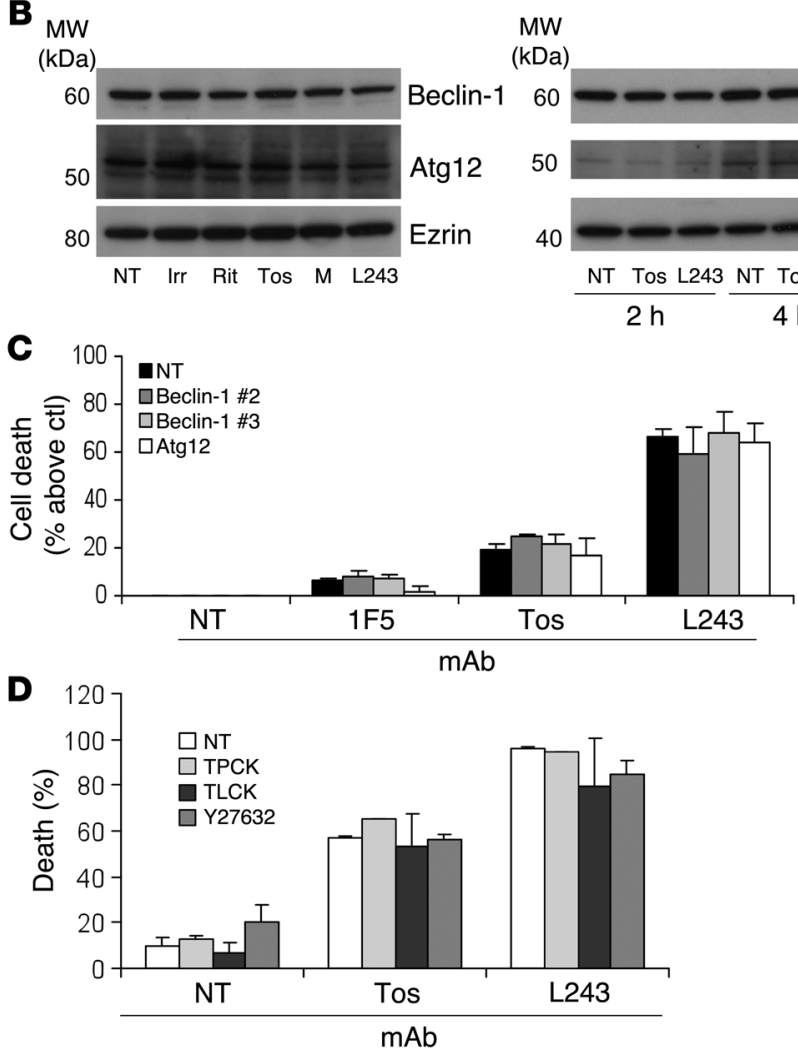

Raji Bcl-2

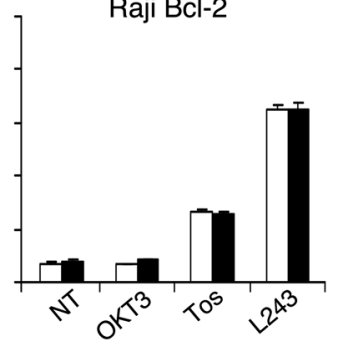

MW

(kDa)

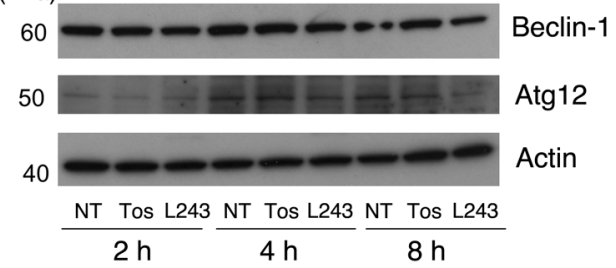

\section{Figure 7}

Cell death evoked by tositumomab and L243 is nonapoptotic and nonautophagic. (A) Raji cells, either WT or overexpressing $\mathrm{Bcl}-2$, were treated with $\mathrm{mAbs}(10 \mu \mathrm{g} / \mathrm{ml})$ or mitoxantrone $(1 \mu \mathrm{g} / \mathrm{ml})$ in the presence (black columns) or absence (white columns) of the pan-caspase inhibitor QVD-OPH (20 $\mu \mathrm{M})$ as indicated for 24 hours and then assessed for cell death as before. (B) Raji cells were treated for 24 hours (left panel) with various $\mathrm{mAbs}(10 \mu \mathrm{g} / \mathrm{ml})$ or treated for 2 hours, 4 hours, or 8 hours with various mAbs (right panel) and then assessed by Western blot for the expression of Beclin-1, Atg12, ezrin, or actin (the latter 2 as loading controls). Irr, irrelevant treated; M, M15/8. (C) Raji cells were nucleofected with siRNA to Atg12 or Beclin-1 and then incubated for 24 hours. Subsequently, cells were treated with $\mathrm{mAbs}(10 \mu \mathrm{g} / \mathrm{ml})$ and assessed for cell death as previously described. The degree of knockdown was verified 48 hours after nucleofection by Western blot (inset). B2 and $\mathrm{B} 3$ refer to 2 different targeting regions of Beclin-1. (D) Raji cells were preincubated with TPCK, trypsin-like serine proteases (TLCK), or Y27632 $(20 \mu \mathrm{M})$ before being treated with tositumomab or L243; cell death was measured 24 hours later. We performed similar experiments with inhibitors over a 50 -fold dilution range from 1 to $50 \mu \mathrm{M}$ with identical results.
Therefore, to characterize the involvement of lysosomes in the cell death induced by tositumomab or L243, we assessed these characteristics in LysoTracker-labeled (27) Raji and SU-DHL4 cells. These experiments revealed that LysoTracker fluorescence increased within 1 hour after addition of $\mathrm{mAbs}$ and reached its maximum within 4 hours in cells treated with tositumomab or L243 mAbs (Figure 9A). Although the increase in fluorescence could reflect an elevation in LysoTracker accumulation due to an increased $\mathrm{pH}$ gradient, we believe that it reflects an enlargement in the lysosomal compartment in these cells. The increase in LysoTracker fluorescence was followed by the appearance of a LysoTracker-low fraction in a proportion of cells after 4 hours, which suggests that the initial swelling of lysosomes is followed by the collapse of this compartment. Fluorescence microscopy of LysoTracker-labeled Ab-treated cells undergoing HA also revealed the presence of highly enlarged lysosomes often present within aggregated cells. (Figure 9A). To directly correlate the lysosomal changes with cell death, we performed dual labeling of cells with LysoTracker and annexin V-Cy5.5 and analyzed cells after treatment with mAbs (Figure 9B). As expected, the loss of lysosomal labeling with LysoTracker was coincident with annexin $\mathrm{V}$ staining, indicating that cell death is directly related to this phenomenon. The degree of LMP after treatment with tositumomab or L243 mAbs was then assessed using a method modified from that used previously (28). In brief, cells were first treated with acridine orange to label lysosomes and then washed prior to addition of mAbs. Acridine orange at acidic $\mathrm{pH}$ (for example in lysosomes) fluoresces red, whereas its leakage from lysosomes into the more neutral $\mathrm{pH}$ of the cytosol results in green fluorescence, indicating an increase in LMP. Increased green fluorescence (FL1) was observed in approximately $30 \%$ of cells 4 hours after tositumomab treatment (Figure 9C). This increase was followed by a reduction in green fluorescence due to subsequent permeabilization of the plasma membrane and leakage of the cytoplasmic content. The latter was verified by fluorescence microscopy, in which cells with nonfluorescent/destained cytoplasm were observed after tositumomab (Figure 9D) or L243 mAb treatment (data not shown). To determine whether lysosomes were releasing their contents in the cell, we performed fluorescent immunohistochemical staining for cathepsin B, a classical lysosomal component. These experiments revealed a substantial increase in cathepsin B staining throughout the cytosol and particularly at the intercellular junctions (Figure 9E). Further investigation using double staining for cathepsin B and lysosomal- 

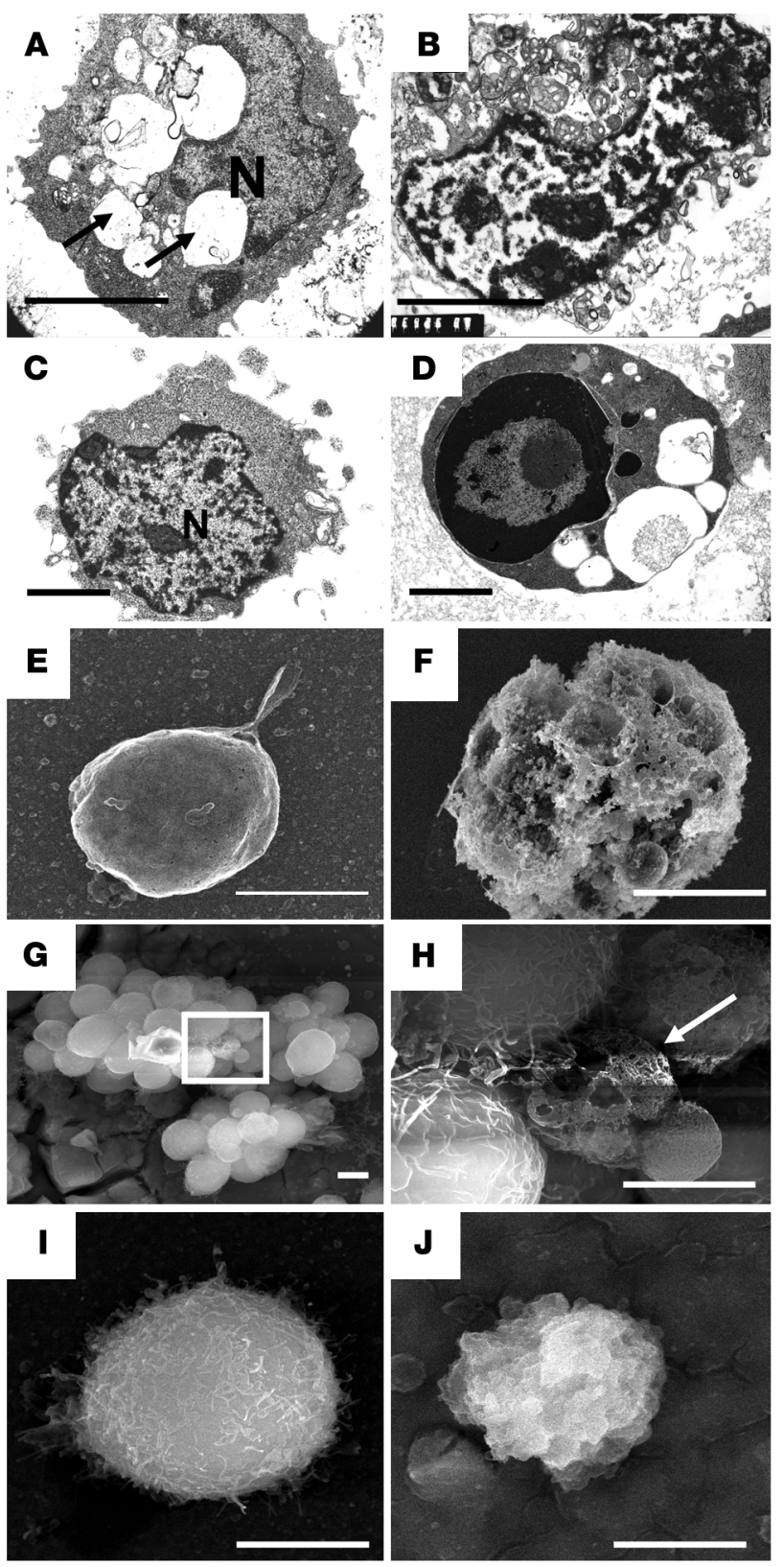

associated membrane protein 1 (LAMP-1) confirmed this observation and in addition revealed that the LAMP-1-positive lysosomes appear to redistribute to the cellular periphery (Figure 9E and inset). The importance of cathepsin B in the cell death process was subsequently confirmed using a specific inhibitor that virtually ablated tositumomab-induced cell death and markedly inhibited the cell death induced by L243 mAbs (Figure 9F).

To further confirm the involvement of lysosomes in this death process, we utilized the well-characterized inhibitor of vacuolar ATPases (V-ATPases), concanamycin A. V-ATPases are proton pumps that serve to energize intracellular membranous vesicles such as lysosomes. Concanamycin A and the structurally related bafilomycin A1 are able to inhibit the acidification of organelles, interfere with protein trafficking (29), and prevent cytotoxicity elicited through perforin and/or Fas (30). Here, we determined whether

\section{Figure 8}

Morphological pattern of cell death induced by anti-CD20 mAb tositumomab. (A and B) Gross vacuolization of cytoplasm (arrows) with relatively intact nuclei $(N)$ is a typical TEM pattern of cell death in the presence of anti-CD20 (Tos) (A) and anti-HLA-DR (L243) Abs (B). (C) Morphology of control, nontreated cell and (D) classical apoptosis. (E-J) Scanning EM figures of tositumomab-treated Raji cells. Scale bars: $10 \mu \mathrm{m}(\mathbf{A}, \mathbf{B}$, and $\mathbf{E}-\mathbf{J}) ; 5 \mu \mathrm{m}$ (C and $\mathbf{D})$. (E) Loss of microvilli was a typical feature of tositumomab-treated cells at the 4-hour time point. (F) Complete permeabilization and decomposition of cytoplasm was observed at 24 hours after treatment $(10 \mu \mathrm{g} / \mathrm{ml})$. (G) HA of Raji cells following treatment with tositumomab $(10 \mu \mathrm{g} / \mathrm{ml})$. (H) Section in the square from $\mathbf{G}$ enlarged. Arrow shows nonapoptotic cell death of aggregated cell. (I) Nontreated cell and (J) morphological control of apoptosis after treatment with cycloheximide for 24 hours $(50 \mu \mathrm{g} / \mathrm{ml})$.

they could block cell death evoked by mAb engagement of CD20 or HLA-DR. Low, submicromolar concentrations of concanamycin A, which were nontoxic and thought to be specific for the V-ATPase, were able to markedly reduce the death of Raji cells following treatment with either tositumomab or L243 and in a dose-dependent manner (Figure 10A). Similar results were generated with bafilomycin A1, demonstrating a clear role for acidic vacuoles (likely lysosomes) in the death process (Figure 10B). Importantly, these inhibitors were able to prevent death of $\mathrm{mAb}$-treated cells for at least 72 hours as demonstrated using sodium 3'-[1-(phenylaminocarbonyl)-3,4-tetrazolium]-bis (4-methoxy-6-nitro) benzene sulfonic acid hydrate (XTT) assays (Figure 10C). In addition, we assessed the effect of concanamycin and bafilomycin A1 on the lysosomal volume in cells treated with mAbs. Figure 10D demonstrates that both concanamycin and bafilomycin A1 reduce the increase in lysosomal volume triggered by tositumomab and L243 mAbs.

\section{Discussion}

The last decade has seen a considerable expansion in the number of mAbs available for the treatment of malignant and autoimmune disease (2). We have previously speculated that "signaling mAbs" provide a selective advantage compared with other $\mathrm{mAb}$ specificities, potentially by eliciting direct cell death programs in the target cells (3). Here, we examined the effects of treating cells with mAbs directed against 2 surface targets, namely CD20 and HLA-DR, that have been developed as anticancer therapeutics in the clinic.

In this study, we have demonstrated that ligating CD20 and HLA-DR (but not other B cell surface antigens, e.g., CD37, CD38) on the surface of malignant B cells (including primary CLL samples) with certain mAbs induced strong HA and subsequent cell death. Two important points arise from this observation, namely, (a) only certain cell-surface targets are capable of evoking these types of responses and (b) the fine binding specificity of the mAbs is critical. Specifically, only type II and not type I anti-CD20 mAbs evoke strong HA (and cell death) while only anti-HLA-DR efficiently perform a similar function for those mAbs targeting HLA molecules (mAbs targeting HLA-DP, HLA-DQ, and pan-HLA do not). Establishing the link between the type/location of binding employed by the different mAbs and their subsequent effects on HA and cell death is the subject of our ongoing research.

The engagement of HLA-DR, CD38, CD40, and other B cell surface antigens, including CD19, CD20, CD21, CD22, and CD23, has previously been shown to induce rapid HA of $B$ cell lines (10). The $\mathrm{HA}$ was not the result of $\mathrm{mAb}$-mediated aggregation and required 
A
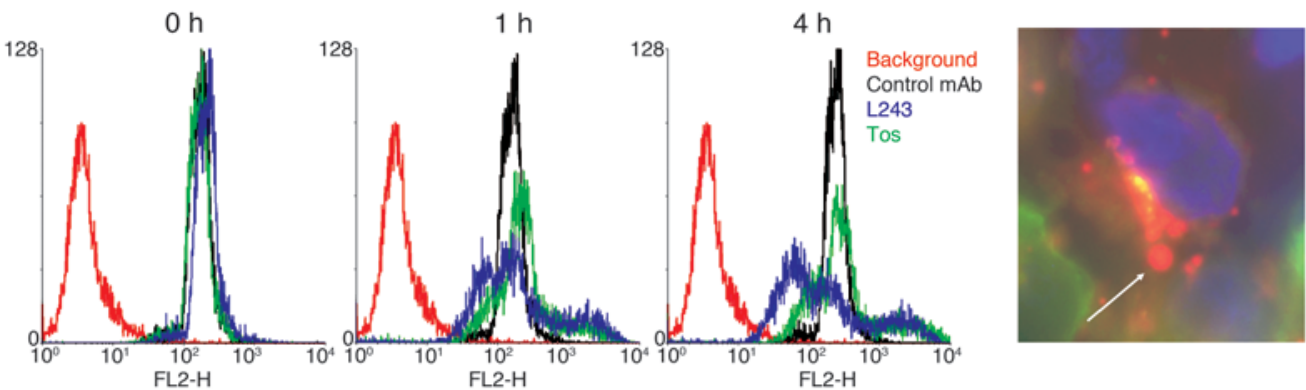

B
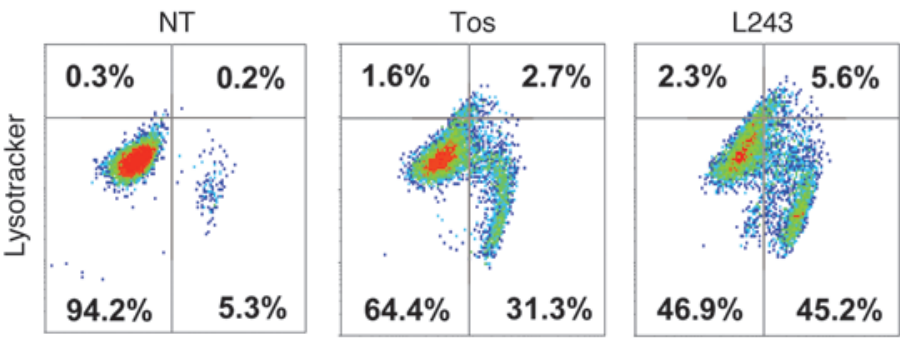

Annexin V Cy5.5
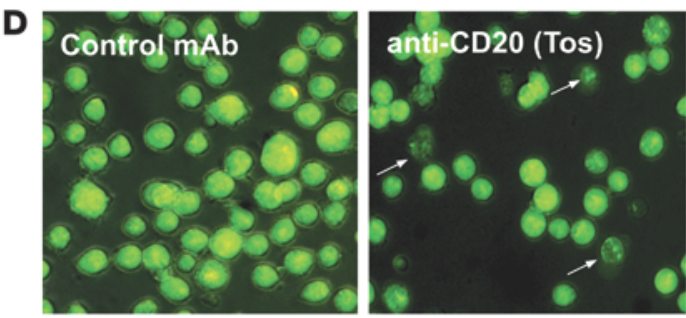

E
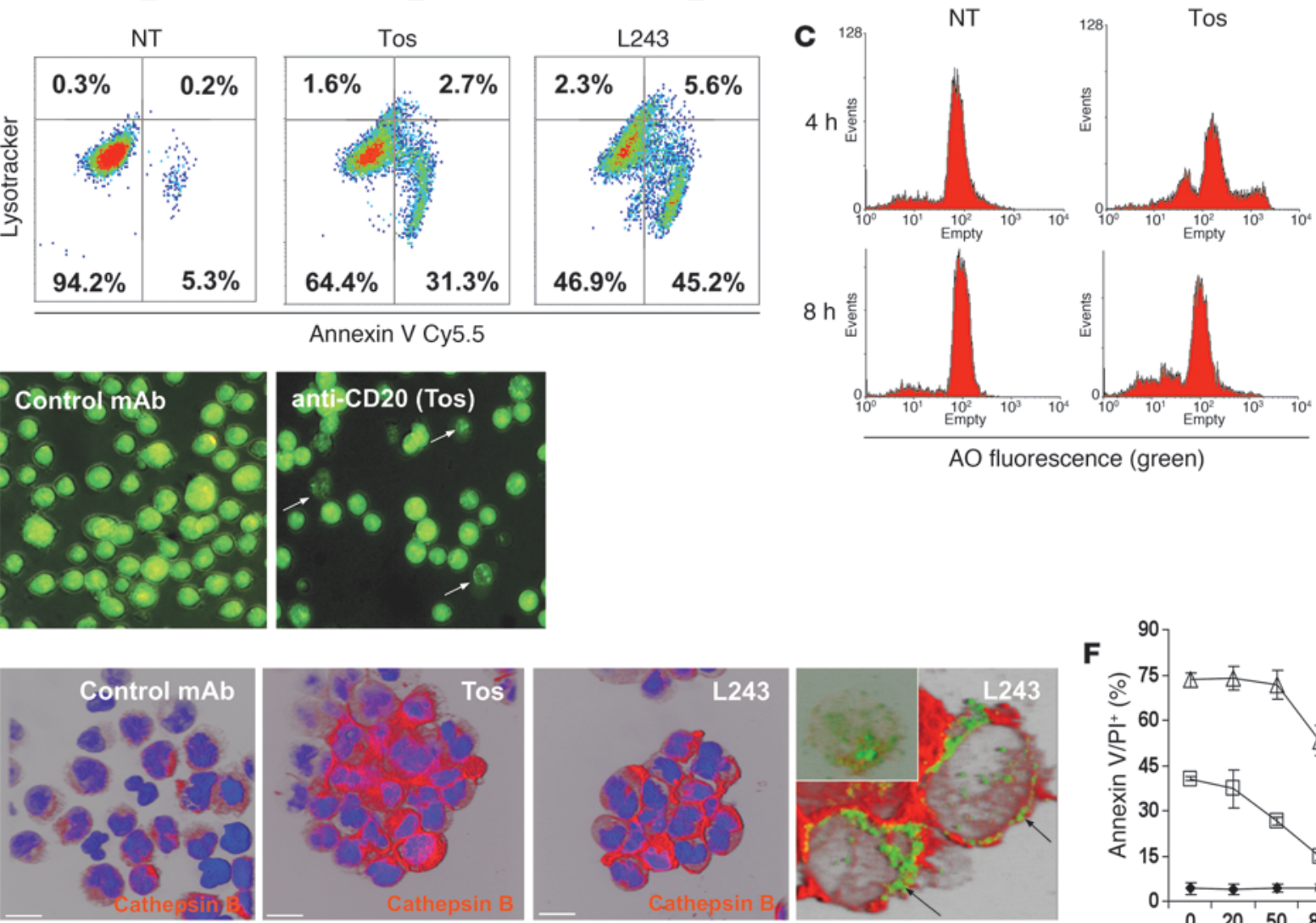

$\mathrm{AO}$ fluorescence (green)
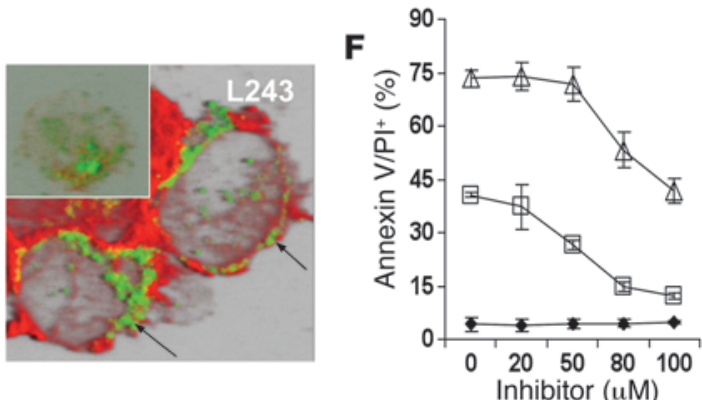

\section{Figure 9}

Involvement of lysosomes in anti-CD20 and anti-HLA-DR-induced cell death. (A) Detection of total lysosomal volume in cells treated with mAbs. Cells were incubated with anti-CD20 mAb tositumomab or anti-HLA-DR mAb L243 as described previously (10 $\mu \mathrm{g} / \mathrm{ml})$. After that, cells were labeled with LysoTracker red and the volume of the lysosomal compartment measured by flow cytometry and fluorescence microscopy after 1 hour or 4 hours. Insert: L243-treated cell displaying a single large lysosome. (B) Relationship between lysosomal volume and cell death. Cells were treated as in A and subsequently, after 4 hours, costained with annexin V-Cy5.5 and assessed by 2-channel flow cytometry. (C) Detection of lysosomal membrane permeabilization followed by permeabilization of cytoplasm. Cells were treated with mAbs as described above and then stained with acridine orange (AO). The relative increase or decrease in FL1 fluorescence was assessed by flow cytometry. (D) Permeabilization of plasma membrane as detected by destained cytoplasm (arrows) of tositumomab-treated cells. Cells were stained with AO as described and then assessed by fluorescence microscopy. Original magnification, $\times 40$. (E) Confocal microscopy of cathepsin B staining (red) 4 hours after treatment with tositumomab or L243 mAbs. DNA was counterstained with DAPI (blue). Bottom right-hand panel shows L243-treated cells costained with cathepsin B (red) and LAMP-1 (green) with control-treated cells shown in the insert. A gross increase in the cathepsin B signal and peripheral localization of LAMP-1 (arrows) was observed. Scale bars: $20 \mu \mathrm{m}$. (F) The impact of cathepsin inhibitor III on cell death induced by tositumomab and L243. Cells were pretreated with different concentrations of inhibitor, and cell death (percentage of annexin V-FITC/PI-positive cells) was assessed 4 hours after treatment. Data represent the average of 3 independent experiments + SEM.

cellular energy and an intact cytoskeleton, but not new mRNA or protein synthesis (10). Furthermore, anti-HLA-DR- and antiCD20 mAb-induced adhesion was lymphocyte function-associated antigen-1 (LFA-1) independent (10) and was mediated by an as-yet-unidentified adhesion receptor(s). With respect to HA, it is particularly noteworthy that both CD20 and MHC class II antigens are localized to microvilli and constitutively associated with membrane rafts $(16,17)$ that in turn are required for the expres- 

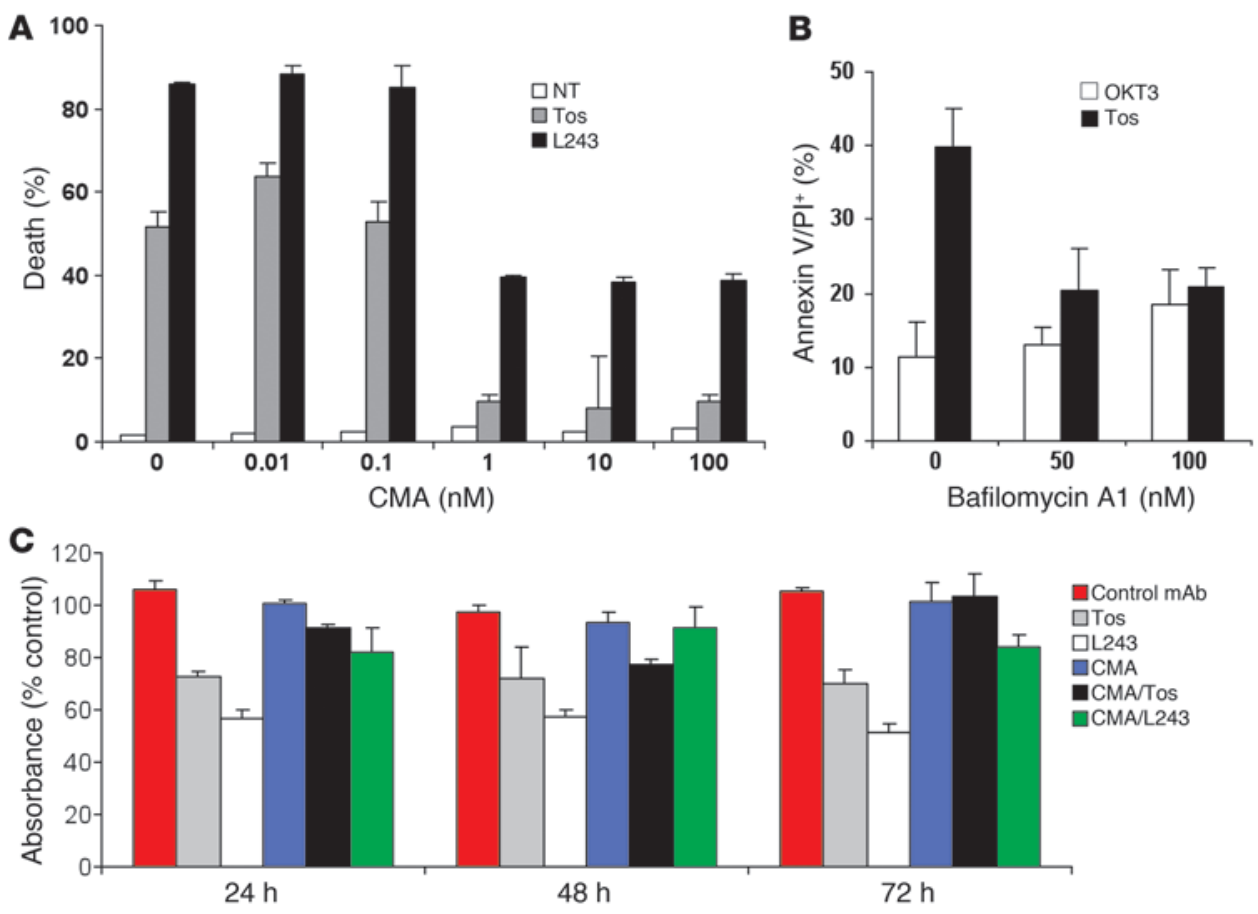

D
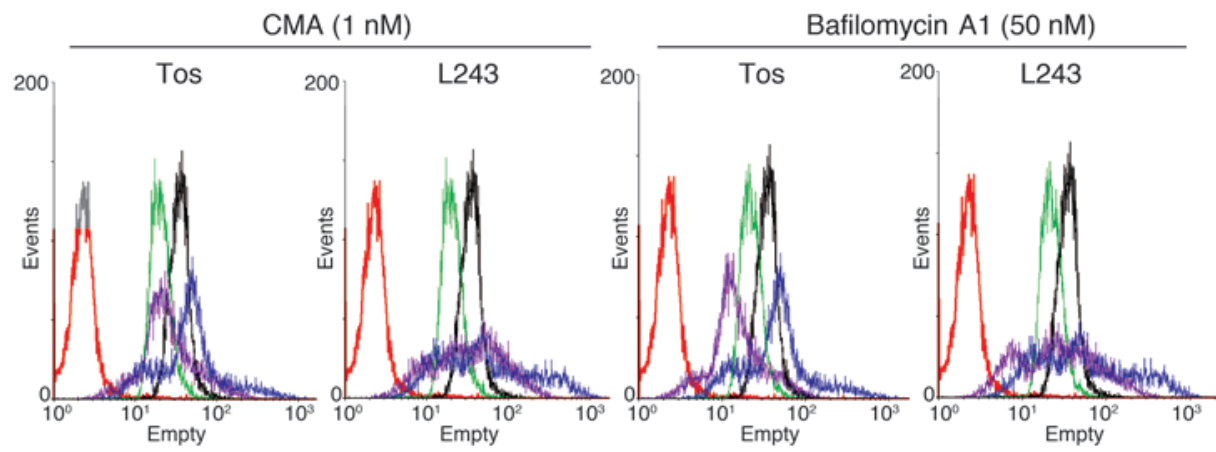

Figure 10

Lysosome-mediated cell death evoked by tositumomab and L243 is blocked by inhibitors of V-ATPase. Raji cells were preincubated with concanamycin A (CMA) $(0.01-100 \mathrm{nM})(\mathbf{A})$ or bafilomycin A1 (0-100 $\mathrm{nM})(B)$ for 30 minutes before being treated with tositumomab or L243 $(10 \mu \mathrm{g} / \mathrm{ml})$. Cell death (AnV/PI+ cells) was measured 24 (A) or 4 (B) hours after treatment. (C) Inhibition of lysosomal V-type ATPase protects cells from long-term cytotoxicity induced by anti-CD20 and HLA-DR mAbs. Cells were pretreated with lysosomal V-type ATPase inhibitor concanamycin A $(0.1 \mathrm{nM})$ for 45 minutes, and then mAbs were added. Cell viability was assessed 24,48 , and 72 hours later using an XTT assay (Roche). The plot represents mean metabolic activity relative to nontreated control from 2 independent experiments in 3 technical replicates. Data are shown as mean + SEM. (D) Impact of vATPase inhibitors on the volume of the cellular lysosomal compartment. Cells were incubated with concanamycin A ( $1 \mathrm{nM})$ or bafilomycin A1 $(50 \mathrm{nM})$ for 30 minutes, and then mAbs were added $(10 \mu \mathrm{g} / \mathrm{ml})$. Subsequently, cells were labeled with LysoTracker red for 1 hour as described in Methods. Volume of lysosomal compartment was measured by flow cytometry as the magnitude of fluorescence in the FL2 channel. Level of fluorescence of Raji cells not labeled with LysoTracker (red histograms) was used as the reference control. Histograms represent FL2 fluorescence values of LysoTracker-labeled control cells with (green) or without (black) the indicated vATPase inhibitors as well as Ab-treated cells with (purple) or without (blue) vATPase inhibitors. detergent-insoluble rafts and attenuated both calcium mobilization and cell death induced by another anti-CD20 Ab, rituximab (31). The central nature of the adhesion to the cell death process was further highlighted in experiments in which adhesion was prevented using agarose, such that cell death with either tositumomab or L243 was greatly reduced.

At the cell-to-cell contact areas, we observed an enrichment of mitochondria in cells undergoing HA. Interestingly, this mitochondrial translocation occurred much later (hours after addition of mAbs) than the cholesteroldependent HA (minutes) and polarization of cellular actin (tens of minutes). This temporal separation may suggest 2 stages of mAb-triggered cellular aggregation at which the initial microvilli and raft-driven adhesion are followed by a stronger aggregation of cells mediated by relocalized actin supported by energy from grouping mitochondria. In that context, junctional actin might initially serve to maintain the cellular organelles within the intercellular contact site. In support of this hypothesis, emerging evidence indicates that while microtubules are important factors for mitochondrial transport, the actin cytoskeleton is required for immobilization of these organelles at the cell cortex $(32,33)$. The latter is in good agreement with our data showing that translocation of polarized mitochondria toward the cell-to-cell contact area was grossly impaired when cells were treated with the actin polymerization inhibitor latrunculin B. Although currently the function of cortical immobilization of mitochondria is not well understood, it is believed that these immobilization events are critical for localization of mitochondria at sites of high ATP utision of microvilli on the cell surface. Therefore, we postulated that extraction of plasma membrane cholesterol would significantly impair the intercellular interaction induced by the mAbs studied. Indeed, HA and cell death were inhibited if cells were pretreated with MCD. In that context, it is notable that cholesterol depletion prevented the association of hyper-cross-linked CD20 with lization (34). In our system, only mitochondria having high membrane potential are relocalized toward the intercellular contact area, suggesting that these areas are the sites of high ATP use.

In addition to tight HA and formation of transient cytoplasmic bridges, we also observed clear evidence of membrane exchange between the interacting cells after treatment with both mAbs. 
Previously, a number of reports have demonstrated that certain cell types, including pheochromocytoma (35), DCs (36-38), T cells (39), natural killer cells (40), and B cell lymphoma (41), have the capacity to transfer membrane and cellular components. In the case of B cell lymphomas, spontaneous membrane exchange is thought to be due to transfer of membrane components during direct cell contact (41) in processes that are enhanced by BCR cross-linking. However, using costaining with annexin V-Cy5.5, we revealed that cells that had undergone membrane exchange were no more likely to die than those that had not, indicating that this is probably an epiphenomenon of closely attached cells rather than a process central to the mechanism of cell death.

Following HA, we observed the induction of nonapoptotic cell death with both tositumomab and L243 mAbs. We previously reported that cell death triggered by tositumomab was not accompanied by DNA fragmentation and was independent of caspase activation, characteristic of classical apoptosis $(5,18)$. These observations were confirmed and extended here, with ultrastructural studies using transmission and scanning EM indicating that the mode of cell death evoked by both mAb specificities strongly resembles "cytoplasmic cell death," as described previously (21-23). An equivalent mode of cell death was also evoked by L243 mAbs, in which both overexpression of Bcl-2 and preincubation with QVDOPH did not have any impact on the extent of cell death.

The HLA-DR molecule is an important component of the immune system, and its engagement on several cell types leads to various cellular events that modulate cell function. For example, signaling via this molecule can lead to rapid B cell death $(42,43)$ that is independent of caspase activation (43-45). In agreement with our data, this cell death involves the integrity of the cytoskeleton and is independent of the LFA-1 system.

It has previously been established that lysosomes control cell death at several levels. A limited release of lysosomal contents to the cytoplasm triggers apoptosis or apoptosis-like cell death, whereas generalized lysosomal rupture results in rapid cellular necrosis (reviewed in ref. 24). In response to endogenous or exogenous stress, LMP can occur, leading to the release of catabolic hydrolases that can mediate caspase-independent cell death or necrosis following high levels of LMP. In accordance with this concept, we observed generalized swelling and rupture of the lysosomal compartment after ligation of CD20 and HLA-DR with mAbs, resulting in release of cathepsin $\mathrm{B}$, leading to a caspase- and Bcl-2independent cell death. Furthermore, lysosomes (stained with LAMP-1) were observed at the cell-cell junctions, with cathepsin staining evident at the cell-cell interface, indicating a relocation and dispersal of lysosomal contents at the cell-cell juncture. Linked to the observation that cell-cell contact/proximity is critical for cell death, these data indicate that high local concentrations of cathepsin B close to intercellular junctions are responsible for the subsequent cell death.

In keeping with these observations, both swelling of lysosomal compartment and cell death could be inhibited with V-ATPase inhibitors concanamycin A and bafilomycin A1, with cell death also blocked by cathepsin inhibitor III. Interestingly, although bafilomycin A1 is a commonly used inhibitor of autophagy, its ability to inhibit anti-CD20-induced cell death was not dependent on the key regulator of autophagy, Beclin-1. This suggests that V-ATPase has a pleiotropic role in nonapoptotic cell death modalities and that its pharmacological inhibition does not necessarily exclude forms of nonapoptotic death other than autophagy. More- over, in our system, V-ATPase appeared to be important for HA of cells, which, to our knowledge, has not been reported previously.

Both mAbs evoked nonapoptotic cell death, as judged by the detailed ultrastructural analysis described above. Inhibition of apoptosis is considered an important factor in the treatment resistance of tumor cells. Therefore, the ability of tositumomab and HLA-DR mAbs such as L243 to bypass apoptotic regulation may be a critical factor in their mode of action. This independence from mitochondrial and death receptor signaling pathways potentially facilitates use of these reagents for treatment of tumors with impaired apoptotic machinery that have become resistant to conventional cytotoxic chemotherapy.

In summary, we have shown for what we believe is the first time that HA and cell death induced by 2 clinically relevant mAbs are interrelated. Treatment with tositumomab and L243 mAbs accelerated the rate of membrane exchange between apposing cells in a process independent of $\mathrm{Fc}-\mathrm{Fc} \gamma \mathrm{R}$ interactions. Furthermore, adhesion and death were both dependent upon redistribution of actin filaments, with the latter resulting from lysosomal enlargement and leakage. The loss of plasma membrane integrity was independent of Bcl-2 control, but correlated with release of cathepsin B, which coupled to the observed cell death pathway involved, indicates that these mAbs are able to bypass the stunted apoptotic machinery in tumor cells resistant to cytotoxic chemotherapy and radiotherapy (18). Together, these data provide fresh insight into how these mAbs may elicit antitumor responses and provide information on a new cell death pathway that is potentially exploitable with novel therapeutics in the clinic.

\section{Methods}

Cell lines and reagents. Human cell lines (Raji and SU-DHL4) were obtained from the European Collection of Cell Cultures (ECACC). Raji cells expressing Bcl-2 were produced previously (18). RJ.2.2.5 cells are a Raji variant that lack HLA expression (46) and were a gift from Roberto Accolla (University of Insubria, Varese, Italy). Cell lines were maintained in antibiotic-free RPMI 1640 medium with FCS (10\%; Sigma-Aldrich), glutamine ( $2 \mathrm{mM}$; Gibco, Invitrogen), and sodium pyruvate $(1 \%)$ at $37^{\circ} \mathrm{C}, 5 \% \mathrm{CO}_{2}$. Jasplakinolide, cytochalasin D, latrunculin B, concanamycin A, LY294002 and Y27632 Cathepsin Inhibitor III, and anti-cathepsin B Abs were purchased from Calbiochem. MCD, bafilomycin A1, and mitoxantrone were purchased from Sigma-Aldrich. Abs directed to Atg12, Beclin-1, and ezrin were purchased from Cell Signaling Technology. Abs to LC3 were a gift from P. Townsend (University of Southampton, Southampton, United Kingdom). Abs to actin were from Sigma-Aldrich. QVD-OPH was purchased from MP. SYTOX Green stain was purchased from Invitrogen.

Abs. Tositumomab (B1; GlaxoSmithKline), 1F5, WR17 (mouse antihuman CD37), L243 (anti-HLA-DR), and OKT3 (anti-CD3) are all of the $\mathrm{mIgG2a}$ isotype and have been described previously (5). Rituximab (antiCD20; Roche) and its murine IgG2a variant were provided by Luke Nolan (University of Southampton) or produced in-house, respectively, as detailed previously (8). M15/8 (anti-IgM), AT13/5 (CD38), F3.3 (pan HLA), A9-1 (pan HLA), B7-2.1 (HLA-DP), and L2 (HLA-DQ) were all derived in-house, obtained from ATCC, or provided through HLDA Workshop 8. IgG was produced from supernatant secreted by hybridomas in tissue culture and purified in-house using protein A columns (GE Healthcare). $\mathrm{F}(\mathrm{ab}){ }_{2}$ fragments of Abs were prepared by trypsin digestion as detailed previously (47).

Clinical samples. Samples were prepared and provided by Ian Mockridge (Molecular Immunology Group, Southampton General Hospital, Southampton, United Kingdom) and Isla Wheatley (Experimental Cancer Medicine Centre, Southampton, United Kingdom). Ethical approval for 
the use of clinical samples was obtained by the Southampton University Hospital National Health Service (NHS) Trust from the Southampton and South West Hampshire Research Ethics Committee. Informed consent was provided in accordance with the Declaration of Helsinki. Blood was obtained from patients with typical CLL who attended the hematology outpatient clinics at Royal Bournemouth Hospital (Bournemouth, United Kingdom), Hammersmith Hospital (London, United Kingdom), Leicester Royal Infirmary (Leicester, United Kingdom), Portsmouth Hospital (Portsmouth, United Kingdom), Southampton General Hospital, and the Royal Wolverhampton Hospitals NHS Trust (Wolverhampton, United Kingdom). PBMCs were isolated by Lymphoprep gradient centrifugation (Axis-Shield), washed, and cryopreserved in RPMI 1640 medium supplemented with 10\% DMSO and 15\% FCS. CLL samples were thawed in complete RPMI 1640 medium containing 10\% FCS, 2 mM glutamine, and 1\% sodium pyruvate. Cells were pelleted by centrifugation, resuspended in complete medium, and counted prior to use.

Western blotting. Cell lysates were prepared in PhosphoSafe lysis solution (Merck). Samples were then separated by SDS-PAGE and proteins transferred immediately onto PVDF membrane (HYBOND; Amersham Pharmacia Biotech) using a semi-dry transfer system (TE 22 system; Hoeffer, Amersham Pharmacia Biotech). Membranes were blocked with 5\% nonfat dried milk, incubated with the appropriately diluted primary Abs, washed, incubated with horseradish peroxidase-conjugated anti-rabbit or antimouse IgG (Sigma-Aldrich), and visualized by ECL (Amersham Pharmacia Biotech) and exposure to light-sensitive film (Hyperfilm ECL, Amersham Pharmacia Biotech).

Cholesterol modulation. For cholesterol depletion, cells were treated in RPMI containing $10 \mathrm{mM} \mathrm{MCD}$ (Sigma-Aldrich) for 30 minutes at $37^{\circ} \mathrm{C}$. For cholesterol repletion, cholesterol-depleted cells were washed and treated in RPMI containing MCD/cholesterol complex (1.4 mm cholesterol in 10 $\mathrm{mm} \mathrm{MCD}$ ) (Sigma-Aldrich) for 30 minutes at $37^{\circ} \mathrm{C}$, followed by washing.

CTB staining. Control and MCD-treated Raji cells were stained with FITC-conjugated CTB (CTB-FITC; Sigma-Aldrich) in PBS at $4^{\circ} \mathrm{C}$ for 30 minutes, washed, and then analyzed by flow cytometry (BD) and WinMDI software (version 2.8; Joseph Trotter, Scripps Research Institute, La Jolla, California, USA).

Light microscopy for measuring HA. HA was assessed by adding mAbs (5-10 $\mu \mathrm{g} / \mathrm{ml}$ ) to cells in flat-bottomed plastic plates (Nunc) and then examining them 4 to 24 hours later. Cells were viewed with an Olympus CKX21 inverted microscope (Olympus) using a $\times 10$ or $\times 20 / 0.25 \mathrm{PH}$ lens. Images were acquired using a CCL2 digital cooled camera (Olympus) and were processed with Cell B (Olympus Soft Imaging Solutions) and Adobe Photoshop, version CS2, software.

Agarose embedding and assessment of cell death with SYTOX Green dye. After $\mathrm{mAb}$ treatment $(10 \mu \mathrm{g} / \mathrm{ml})$, the cell suspension was immediately mixed 1:1 with $2 \%$ low-melting point agarose at $37^{\circ} \mathrm{C}$ in complete medium containing appropriate therapeutic $\mathrm{Abs}(10 \mu \mathrm{g} / \mathrm{ml})$ and SYTOX Green DNA dye $(1 \mu \mathrm{M})$. The resulting mixture, containing $1 \%$ agarose, was allowed to set on ice for 30 seconds, and then HA and SYTOX Green fluorescence were assessed under the fluorescence microscope at given time intervals.

TEM. Cells were washed and fixed in $3 \%$ glutaraldehyde (Sigma-Aldrich) before pelleting ( $500 \mathrm{~g}$ for 5 minutes) and fixing at $4{ }^{\circ} \mathrm{C}$ overnight. The fixative was removed and the pellets washed 3 times in PBS prior to pelleting again. Following removal of the supernatant, the cell pellets were post-fixed for 1 hour in 1\% osmium tetroxide (Agar Scientific) in PBS. Fixative was removed and the pellets washed a further 3 times. Cell pellets were dehydrated through a graded series of ethanol. The pellets were then placed in $100 \%$ Acetone (Merck), disaggregated, embedded with Agar 100 resin (Agar Scientific), placed into prelabeled BEAM capsules (Agar Scientific) filled with fresh $100 \%$ Agar 100, and polymerized at $60^{\circ} \mathrm{C}$ for 48 hours.
The embedded pellets were sectioned (60-nm thick), collected on copper TEM grids (Agar Scientific), and post-stained with uranyl acetate and lead citrate prior to imaging in a JEOL1220 TEM at $120 \mathrm{kV}$ (JEOL). Images were acquired digitally with a Gatan Orius side mount CCD camera and Digital Micrograph software, version 1 (Gatan).

Scanning EM. Cells were washed and fixed overnight as detailed above. The cell suspension was spun (128 g for 5 minutes) and the pellet washed 3 times in PBS before mounting onto polylysine-coated $(1 \mathrm{mg} / \mathrm{ml}) 5 \mathrm{~mm}$-silicon chips (Agar Scientific). The chips were removed and placed into $35-\mathrm{mm}$ disposable Petri dishes containing $1 \%$ osmium tetroxide in PBS and post-fixed for 1 hour. Chips were then washed in PBS, 3 times for 5 minutes each time, before being dehydrated through an ethanol series and then critical-point dried in a BAL-TEC CPD030 critical point dryer using $\mathrm{CO}_{2}$ as the transition fluid. The chips were then coated with $8 \mathrm{~nm}$ of chromium in an Edwards Auto 308 Cryo Sputter Coater (BOC Edwards) and subsequently imaged in a lens DS130 FESEM (ABT) at $30 \mathrm{kV}$. Images were acquired digitally using an iScan Image Capture System (ISS GROUP).

Lysosomal volume and permeability assessment. To assess lysosomal volume, cells were labeled with $75 \mathrm{nM}$ LysoTracker probe (Invitrogen) at different time points after treatment with mAbs. FL2 fluorescence of LysoTrackerlabeled cells was assessed 1 hour after labeling. Unlabeled cells were used as a background control. For double LysoTracker/annexin V staining, cells were incubated with $\mathrm{mAbs}$, labeled with LysoTracker probe, washed, and resuspended in $200 \mu \mathrm{l}$ of annexin V-binding buffer containing $5 \mu \mathrm{l}$ of Cy5.5-labeled annexin V. Two channel flow cytometry of FL2 (LysoTracker) and FL4 (annexin V-Cy5.5) fluorescence was then performed using a FACSCalibur cytometer (BD Biosciences).

For lysosome permeability measurements, cells were incubated with $5 \mu \mathrm{M}$ acridine orange (Molecular Probes; Invitrogen) for 15 minutes at $37^{\circ} \mathrm{C}$ and washed twice with PBS before the indicated treatments or measurements. Acridine orange is a metachromatic fluorochrome and a weak base that exhibits red fluorescence when highly concentrated in acidic lysosomes and green fluorescence when outside lysosomes. Total lysosomal integrity was evaluated by assessing green fluorescence (FL1) by flow cytometry or fluorescence microscopy.

Fluorescence, time lapse, and confocal microscopy. For immunofluorescence and confocal microscopy, harvested cells were cytospun onto poly-L-lysinecoated microscope slides. Samples were fixed in $2 \%$ paraformaldehyde at room temperature for 15 minutes (phalloidin) or in $-20^{\circ} \mathrm{C}$ methanol (cathepsin B and LAMP-1) and rinsed briefly in ice-cold acetone. Slides were washed 3 times for 10 minutes each time and then incubated with appropriate primary Abs for 60 minutes at room temperature or overnight at $4{ }^{\circ} \mathrm{C}$. The primary Abs were diluted in TBS $/ 0.1 \%$ BSA. Subsequently, slides were washed in TBS ( 3 times for 10 minutes each time) and stained using the anti-rabbit Alexa Fluor 488 or Alexa Fluor 594 SFX Kit (Invitrogen) according to the manufacturer's protocol. After 3 washes in TBS $/ 0.05 \%$ Tween-20, DNA was counterstained with 7-aminoactinomycin D or DAPI before mounting in ProLong Gold Antifade Reagent (Invitrogen). Subsequently, cells were assessed using a Zeiss Axiovert $200 \mathrm{M}$ fluorescence microscope equipped with Chroma Sedat excitation filters (402/488/555 $\mathrm{nm}$ ) or a MAG Nipkow Spinning Disk confocal microscope equipped with 405-, 491-, and 561-nm imaging lasers. For time-lapse microscopy, cells were put into thin glass-bottomed Petri dishes in a humid atmosphere at $37^{\circ} \mathrm{C}$ and assessed using a Zeiss 7D time-lapse microscope equipped with a CoolSNAP HQ camera and MetaMorph software, version 1. Images were taken every 5 minutes.

Cell transfection. Raji cells were transfected with pAcGFP-actin (Clontech) using the Amaxa Nucleofection Device (Buffer T; Programme G16) according to the manufacturer's instructions. Transfection efficiency was 
approximately $30 \%$, and cell death rate was approximately $5 \%-10 \%$ above that of controls 24 hours after transfection. 24 hours later, cells were transferred to 96-well plates and selected in the presence of G418 $(2 \mathrm{mg} / \mathrm{ml}$; Invitrogen). Two weeks later, wells were screened for GFP expression by flow cytometry; positive wells were cloned and then expanded.

FRAP analysis. Samples for FRAP experiments were prepared by placing a droplet of GFP-actin-labeled Raji cells into IWAKI glass-bottom Petri dishes. The optical plane was set to the middle of the sample. Before bleaching, a stack of 10 images was scanned using an Olympus BX71 microscope to record the prebleach situation. Bleaching was carried out with the Argon laser at a wavelength of $488 \mathrm{~nm}$ for 1 seconds to the $1 \mu \mathrm{m}$ region of interest within the cytoplasm. The recovery of fluorescence was assessed every 2 seconds for 100 seconds overall.

Statistics. Values were compared using unpaired, 2-tailed Student's $t$ test. $P<0.05$ was considered statistically significant in all calculations.

\section{Acknowledgments}

We are grateful to Roberto Accolla (University of Insubria, Varese, Italy) for the kind gift of RJ.2.2.5 cells and Paul Townsend and
Sarah Bailey (Southampton University School of Medicine, Southampton General Hospital) for assistance with hypoxia experiments. We are also grateful to Ian Mockridge (Molecular Immunology Group, Southampton General Hospital) and Isla Wheatley (Experimental Cancer Medicine Centre [ECMC], Southampton, United Kingdom) for provision and assistance with clinical material and Steve Bagley (University of Manchester, Manchester, United Kingdom) for excellent assistance in imaging studies. This work was supported by fellowships and grants from Leukaemia Research, United Kingdom; Cancer Research, United Kingdom; the Association of International Cancer Research; Tenovus; and ECMC.

Received for publication October 27, 2008, and accepted in revised form May 20, 2009.

Address correspondence to: Mark Cragg, Tenovus Research Lab, Southampton General Hospital, Tremona Road, Southampton SO16 6YD, United Kingdom. Phone: 44-23-80777222 ext. 8056; Fax: 44-23-80704-061; E-mail: msc@soton.ac.uk.
1. Glennie, M.J., and Johnson, P.W. 2000. Clinical trials of antibody therapy. Immunol. Today. 21:403-410.

2. Glennie, M.J., and van de Winkel, J.G. 2003. Renaissance of cancer therapeutic antibodies. Drug Discov. Today. 8:503-510.

3. Cragg, M.S., French, R.R., and Glennie, M.J. 1999. Signaling antibodies in cancer therapy. Curr. Opin. Immunol. 11:541-547.

4. Glennie, M.J., French, R.R., Cragg, M.S., and Taylor, R.P. 2007. Mechanisms of killing by antiCD20 monoclonal antibodies. Mol. Immunol. 44:3823-3837.

5. Chan, H.T., et al. 2003. CD20-induced lymphoma cell death is independent of both caspases and its redistribution into triton X-100 insoluble membrane rafts. Cancer Res. 63:5480-5489.

6. Cragg, M.S., and Glennie, M.J. 2004. Antibody specificity controls in vivo effector mechanisms of anti-CD20 reagents. Blood. 103:2738-2743.

7. Cragg, M.S., et al. 2003. Complement-mediated lysis by anti-CD $20 \mathrm{mAb}$ correlates with segregation into lipid rafts. Blood. 101:1045-1052.

8. Beers, S.A., et al. 2008. Type II (tositumomab) anti-CD20 monoclonal antibody out performs Type I (rituximab-like) reagents in B-cell depletion regardless of complement activation. Blood. 112:4170-4177.

9. Dustin, M.L. 2007. Cell adhesion molecules and actin cytoskeleton at immune synapses and kinapses. Curr. Opin. Cell Biol. 19:529-533.

10. Kansas, G.S., and Tedder, T.F. 1991. Transmembrane signals generated through MHC class II, CD19, CD20, CD39, and CD40 antigens induce LFA-1-dependent and independent adhesion in human B cells through a tyrosine kinase-dependent pathway. J. Immunol. 147:4094-4102.

11. Leverkus, M., et al. 2003. MHC class II-mediated apoptosis in dendritic cells: a role for membraneassociated and mitochondrial signaling pathways. Int. Immunol. 15:993-1006.

12. Cerisano, V., et al. 2004. Molecular mechanisms of CD99-induced caspase-independent cell death and cell-cell adhesion in Ewing's sarcoma cells: actin and zyxin as key intracellular mediators. Oncogene. 23:5664-5674.

13. Kasinrerk, W., Tokrasinwit, N., Moonsom, S., and Stockinger, H. 2000. CD99 monoclonal antibody induce homotypic adhesion of Jurkat cells through protein tyrosine kinase and protein kinase Cdependent pathway. Immunol. Lett. 71:33-41.

14. Cao, L., et al. 1997. Anti-CD53 monoclonal antibody induced LFA-1/ICAM-1-dependent and -independent lymphocyte homotypic cell aggregation. Immunobiology. 197:70-81.

15. Walshe, C.A., et al. 2008. Induction of cytosolic calcium flux by CD20 is dependent upon B Cell antigen receptor signaling. J. Biol. Chem. 283:16971-16984.

16. Greicius, G., et al. 2004. Microvilli structures on B lymphocytes: inducible functional domains? Int. Immunol. 16:353-364.

17. Li, H., et al. 2004. The CD20 calcium channel is localized to microvilli and constitutively associated with membrane rafts: antibody binding increases the affinity of the association through an epitopedependent cross-linking-independent mechanism. J. Biol. Chem. 279:19893-19901.

18. Ivanov, A., Krysov, S., Cragg, M.S., and Illidge, T. 2008. Radiation therapy with tositumomab (B1) anti-CD20 monoclonal antibody initiates extracellular signal-regulated kinase/mitogenactivated protein kinase-dependent cell death that overcomes resistance to apoptosis. Clin. Cancer Res. 14:4925-4934.

19. Melino, G., Knight, R.A., and Nicotera, P. 2005. How many ways to die? How many different models of cell death? Cell Death Differ. 12(Suppl. 2):1457-1462.

20. Bras, M., et al. 2007. Drp1 mediates caspase-independent type III cell death in normal and leukemic cells. Mol. Cell. Biol. 27:7073-7088.

21. Sperandio, S., de Belle, I., and Bredesen, D.E. 2000. An alternative, nonapoptotic form of programmed cell death. Proc. Natl. Acad. Sci. U. S. A. 97:14376-14381.

22. Wang, Y., et al. 2004. An alternative form of paraptosis-like cell death, triggered by TAJ/TROY and enhanced by PDCD5 overexpression. J. Cell Sci. 117:1525-1532.

23. Zhang, C., Xu, Y., Gu, J., and Schlossman, S.F. 1998. A cell surface receptor defined by a $\mathrm{mAb}$ mediates a unique type of cell death similar to oncosis. Proc. Natl. Acad. Sci. U. S. A. 95:6290-6295.

24. Kroemer, G., and Jaattela, M. 2005. Lysosomes and autophagy in cell death control. Nat. Rev. Cancer. 5:886-897.

25. He, J., et al. 2005. Lysosome is a primary organelle in B cell receptor-mediated apoptosis: an indispensable role of Syk in lysosomal function. Genes Cells. 10:23-35.

26. Groth-Pedersen, L., Ostenfeld, M.S., Hoyer-Hansen, M., Nylandsted, J., and Jaattela, M. 2007. Vincristine induces dramatic lysosomal changes and sensitizes cancer cells to lysosome-destabilizing siramesine. Cancer Res. 67:2217-2225.
27. Ono, K., Kim, S.O., and Han, J. 2003. Susceptibility of lysosomes to rupture is a determinant for plasma membrane disruption in tumor necrosis factor alpha-induced cell death. Mol. Cell. Biol. 23:665-676.

28. Nylandsted, J., et al. 2004. Heat shock protein 70 promotes cell survival by inhibiting lysosomal membrane permeabilization. J. Exp. Med. 200:425-435.

29. Muroi, M., Shiragami, N., Nagao, K., Yamasaki, M., and Takatsuki, A. 1993. Folimycin (concanamycin A), a specific inhibitor of V-ATPase, blocks intracellular translocation of the glycoprotein of vesicular stomatitis virus before arrival to the Golgi apparatus. Cell Struct. Funct. 18:139-149.

30. Kataoka, T., et al. 1996. Concanamycin A, a powerful tool for characterization and estimation of contribution of perforin- and Fas-based lytic pathways in cellmediated cytotoxicity. J. Immunol. 156:3678-3686.

31. Unruh, T.L., et al. 2005. Cholesterol depletion inhibits src family kinase-dependent calcium mobilization and apoptosis induced by rituximab crosslinking. Immunology. 116:223-232.

32. Yang, H.C., Palazzo, A., Swayne, T.C., and Pon, L.A. 1999. A retention mechanism for distribution of mitochondria during cell division in budding yeast. Curr. Biol. 9:1111-1114.

33. Van Gestel, K., Kohler, R.H., and Verbelen, J.P. 2002. Plant mitochondria move on F-actin, but their positioning in the cortical cytoplasm depends on both F-actin and microtubules. J. Exp. Bot. 53:659-667.

34. Hollenbeck, P.J., and Saxton, W.M. 2005. The axonal transport of mitochondria. J. Cell Sci. 118:5411-5419.

35. Rustom, A., Saffrich, R., Markovic, I., Walther, P., and Gerdes, H.H. 2004. Nanotubular highways for intercellular organelle transport. Science. 303:1007-1010.

36. Watkins, S.C., and Salter, R.D. 2005. Functional connectivity between immune cells mediated by tunneling nanotubules. Immunity. 23:309-318.

37. Valdez, Y., et al. 2002. Major histocompatibility complex class II presentation of cell-associated antigen is mediated by CD8alpha+ dendritic cells in vivo. J. Exp. Med. 195:683-694.

38. Harshyne, L.A., Watkins, S.C., Gambotto, A., and Barratt-Boyes, S.M. 2001. Dendritic cells acquire antigens from live cells for cross-presentation to CTL. J. Immunol. 166:3717-3723.

39. Nolte-'t Hoen, E.N., et al. 2004. Uptake of membrane molecules from $T$ cells endows antigen-presenting cells with novel functional properties. Eur. J. Immunol. 34:3115-3125.

40. Onfelt, B., Nedvetzki, S., Yanagi, K., and Davis, 
D.M. 2004. Cutting edge: Membrane nanotubes connect immune cells. J. Immunol. 173:1511-1513.

41. Poupot, M., and Fournie, J.J. 2003. Spontaneous membrane transfer through homotypic synapses between lymphoma cells. J. Immunol. 171:2517-2523. 42. Leveille, C., Zekki, H., Al-Daccak, R., and Mourad, W. 1999. CD40- and HLA-DR-mediated cell death pathways share a lot of similarities but differ in their use of ADP-ribosyltransferase activities. Int. Immunol. 11:719-730.
43. Zhang, N., Khawli, L.A., Hu, P., and Epstein, A.L. 2007. Lym-1-induced apoptosis of non-Hodgkin's lymphomas produces regression of transplanted tumors. Cancer Biother. Radiopharm. 22:342-356.

44. Bains, S.K., et al. 2003. Mitochondria control of cell death induced by anti-HLA-DR antibodies. Lenkemia. 17:1357-1365.

45. Carmagnat, M., et al. 2006. Dissociation of caspase-mediated events and programmed cell death induced via HLA-DR in follicular lymphoma. Onco- gene. 25:1914-1921.

46. Accolla, R.S. 1983. Human B cell variants immunoselected against a single Ia antigen subset have lost expression of several Ia antigen subsets. J. Exp. Med. 157:1053-1058.

47. Glennie, M.J., McBride, H.M., Worth, A.T., and Stevenson, G.T. 1987. Preparation and performance of bispecific $\mathrm{F}\left(\mathrm{ab}^{\prime}\right.$ gamma)2 antibody containing thioether-linked Fab' gamma fragments. J. Immunol. 139:2367-2375. 